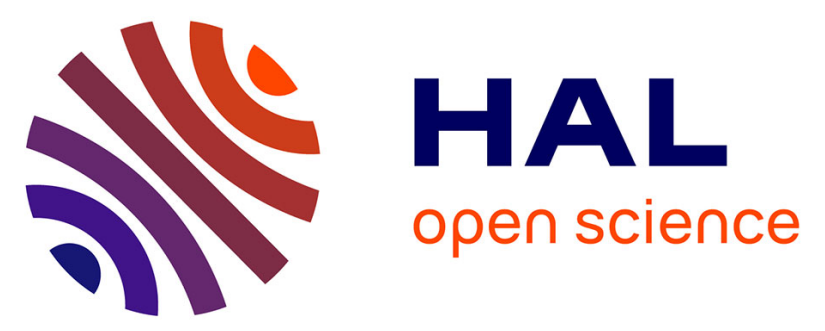

\title{
The Impact of Radial and Non-Radial IMF on the Earth's Magnetopause Size, Shape, and Dawn-Dusk Asymmetry From Global 3D Kinetic Simulations
} Suleiman Baraka, Olivier Le Contel, Lotfi Ben-Jaffel, William Moore

\section{- To cite this version:}

Suleiman Baraka, Olivier Le Contel, Lotfi Ben-Jaffel, William Moore. The Impact of Radial and Non-Radial IMF on the Earth's Magnetopause Size, Shape, and Dawn-Dusk Asymmetry From Global 3D Kinetic Simulations. Journal of Geophysical Research Space Physics, 2021, 126 (10), 10.1029/2021JA029528. hal-03407917

\section{HAL Id: hal-03407917 https://hal.science/hal-03407917}

Submitted on 9 Nov 2021

HAL is a multi-disciplinary open access archive for the deposit and dissemination of scientific research documents, whether they are published or not. The documents may come from teaching and research institutions in France or abroad, or from public or private research centers.
L'archive ouverte pluridisciplinaire HAL, est destinée au dépôt et à la diffusion de documents scientifiques de niveau recherche, publiés ou non, émanant des établissements d'enseignement et de recherche français ou étrangers, des laboratoires publics ou privés. 


\title{
The impact of Radial and Non-Radial IMF on the Earth's Magnetopause Size, Shape, and Dawn-Dusk Asymmetry from Global 3D Kinetic Simulations
}

\author{
Suleiman M Baraka ${ }^{1,3}$, O. Le Contel ${ }^{2}$, L. Ben-Jaffel ${ }^{3}$, W. B. Moore ${ }^{1,4}$ \\ ${ }^{1}$ National Institute of Aerospace, 100 Exploration Way, Hampton, VA 23666 \\ ${ }^{2}$ Laboratoire de Physique des Plasmas, UMR7648, CNRS/Sorbonne Université/Université \\ Paris-Saclay/Observatoire de Paris/Ecole Polytechnique Institut Polytechnique de Paris, Palaiseau, \\ France. \\ ${ }^{3}$ Institut d'Astrophysique de Paris. Sorbonne Universités, UPMC \& CNRS, 98 bis Bd. Arago, F-75014 \\ Paris, France \\ ${ }^{4}$ Hampton University, 154 William R. Harvey Way Hampton, VA 23668
}

\section{Key Points:}

- The magnetopause expansion at the subsolar point for radial and non-radial IMF is partly due to the reduction of the solar wind dynamic pressure caused by backstreaming ions.

- Although backstreaming ions constitute only $16.5 \%$ and $20 \%$ of the solar wind bulk flow for radial and non-radial IMF, they strongly modify the dynamic pressure.

- For both radial and non-radial IMF, the magnetopause is asymmetric, being more extended on the duskside than on the dawnside.

Corresponding author: Suleiman M Baraka, suleiman.baraka@nianet.org

This article has been accepted for publication and ${ }^{-1}$ undergone full peer review but has not been through the copyediting, typesetting, pagination and proofreading process, which may lead to differences between this version and the Version of Record. Please cite this article as doi: 10.1029/2021JA029528. 


\section{Abstract}

The boundary between the solar wind (SW) and the Earth's magnetosphere, the magnetopause (MP), is highly dynamic. Its location and shape depend on SW dynamic pressure and interplanetary magnetic field (IMF) orientation. We use a 3D kinetic Particle-In-Cell code (IAPIC) to simulate an event observed by THEMIS spacecraft on July 16, 2007. We investigate the impact of radial $\left(\theta_{B x}=0^{\circ}\right)$ and non-radial $\left(\theta_{B x}=50^{\circ}\right)$ IMF on the shape and size of Earth's MP for a dipole tilt of $31^{\circ}$ using maximum density gradient and pressure balance methods. Using the Shue model as a reference (MP at $10.3 R_{E}$ ), we find that for non-radial IMF the MP expands by 1.4 and $1.7 R_{E}$ along the the Sun-Earth $(\mathrm{OX})$ and tilted magnetic equatorial (Tilt) axes, respectively, and it expands by 0.5 and $1.6 R_{E}$ for radial IMF along the same respective axes. When the effect of backstreaming ions is removed from the bulk flow, the expansion ranges are 1.0 and $1.3 R_{E}$ and 0.2 , and $1.2 R_{E}$, respectively. It is found that the percentage of backstreaming to bulk flow ions are $16.5 \%$ and $20 \%$ for radial and non-radial IMF. We also show that when the backstreaming ions are not identified, up to $40 \%$ of the observed expansion that is due to backstreaming particles can be inadvertently attributed to a change in the SW upstream properties. Finally, we quantified the temperature anisotropy in the magnetosheath, and observe a strong dawn-dusk asymmetry in the MP location, being more extended on the duskside than on the dawnside.

\section{Plain Language Summary}

The Earth's magnetopause (MP) is a sensitive region where the pressure of the Earth magnetic field balances the shocked solar wind ram and thermal pressures. Accurate space weather monitoring and forecast require an in-depth knowledge of this region and of the physical processes that affect it. We investigate the effects of the orientation of the interplanetary magnetic field (IMF) on the MP size, location, and shape by using a numerical technique (3D kinetic simulation) that uses macro-ions and macro-electrons to mimic the real charged species and explicitly resolves their motion. We are able to extract the shape and location of the MP in three dimensions to estimate the sensitivity of the MP position to 
48 the ambient IMF and solar wind conditions, while our explicit tracking of ion motions allows

49 us to investigate the role of ion species backscattered by the Earth's bow shock and magne-

50 tosheath. We find that when the IMF has a strong component in the Earth-Sun direction

${ }_{51}$ (radial or non-radial IMF), these backscattered ions play an important role in reducing the 52 effective pressure of the incoming solar wind and allowing the MP to expand outward. 


\section{Introduction}

The magnetic fields of planets such as Mercury, Earth, and the giant planets present an obstacle to the supersonic solar wind (SW). As a result, a shock forms and the solar wind is redirected around the obstacle producing a cavity which is called the magnetosphere (e.g., Parks, 1991). The boundary between the shocked SW and the plasma in the magnetosphere is the magnetopause (MP). At the subsolar point, the classical fluid description of the SW stagnation flow derives the location of the magnetopause by the balance between the planetary magnetic field pressure and the dynamic pressure of the SW. Plasma boundary layers form on either side of the magnetopause with the magnetosheath boundary layer (MSBL) on the sunward side and the low-latitude boundary layer (LLBL) on the magnetosphere side. Both layers play an important role in plasma exchange across the magnetopause (e.g., Pi et al., 2018).

The magnetopause structure is significantly influenced by the interplanetary magnetic field (IMF) orientation. While the impact of southward (Yu \& Ridley, 2009; Heikkila, 2011; Tan et al., 2011; Suvorova \& Dmitriev, 2015; Berchem et al., 2016) and northward IMF (Sorathia et al., 2019; Luo et al., 2013; Bobra et al., 2004; Wang et al., 2018) on the dynamics of Earth's magnetosphere have been extensively studied in the last four decades, only recently has attention been focused on radially-dominant IMF conditions, which will be called radial IMF for the remainder of this paper. For most solar wind plasma conditions at the orbital position of planets, bow shocks are collisionless and supercritical shocks, which by definition, reflect and accelerate a fraction of the plasma impinging on them. These backstreaming particles lead to the formation of the ion foreshock region upstream (e.g., Turner et al., 2018, p. 206).

Following early satellite observations (Greenstadt et al., 1968; Asbridge et al., 1968), the idea of an extended foreshock that diverts the solar wind around the magnetosphere and reduces the solar wind dynamic pressure at the subsolar magnetopause was proposed for radial IMF conditions (Fairfield et al., 1990; Merka et al., 2003; Jelínek et al., 2010; 
Suvorova et al., 2010). The distance and shape of the equatorial magnetopause is strongly affected by radial IMF, resulting in a global expansion of the magnetopause (Grygorov et al., 2017). Zhang et al. (2019) found that a dawn-dusk asymmetry exists in the magnetosheath, directly related to the IMF orientation. Evidently, the plasma distribution and the IMF are correlated to these asymmetries which are either generated at the bow shock or inside the magnetosheath itself.

Most magnetopause observations during radial IMF have noticed a large magnetopause expansion that was connected with a significant distortion of the magnetopause surface. Large magnetopause distortion and anomalous sunward magnetosheath flows were reported in one radial IMF event by (Shue et al., 2009). The finding of magnetopause displacement during radial IMF conditions was also documented in a statistical study based on a large set of magnetopause crossings using THEMIS (Dušík et al., 2010) and GOES (Park et al., 2016) data. A systematic increase of observed magnetopause distances for radial IMF was found, ranging from $0.3 R_{E}$ at $90^{\circ}$ cone angle to $\approx 1.7 R_{E}$ at $0^{\circ}$ or $180^{\circ}$ cone angles compared to empirical models. However, Duš́́k et al. (2010); Suvorova and Dmitriev (2015) pointed out a large dispersion in their statistical results attributed to the difficulty to propagate radial IMF to the magnetopause. In contrast, using THEMIS data and empirical models of the MP, Grygorov et al. (2017) concluded that the distance of the equatorial magnetopause is strongly affected by radial IMF, expanding globally and independent of the local time, upstream value of other solar wind parameters or the tilt of the Earth magnetic dipole.

It is interesting to remark that no self-consistent model exists today in the literature that can explain the observed MP displacement or its asymmetry, particularly with the difficulty MHD approaches have to accurately model reflected SW ions in the foreshock region (Sibeck et al., 2001). In a recent study, (Samsonov et al., 2017) used previous statistical results to suggest that the density and velocity in the foreshock region decrease to $\sim 60 \%$ and $\sim 94 \%$ of the undisturbed SW values when the cone angle falls below $50^{\circ}$ causing a drop in the SW dynamic pressure of $\sim 53 \%$ that might cause the MP displacement. In a second step, 
those authors modified the upstream SW parameters in a global MHD model to take these foreshock effects into account, which helped them predict MP distances during non-radial IMF intervals close to those observed by THEMIS. More precisely, using time dependent SW conditions provided by ACE and WIND measurements in their simulations, they applied $\mathrm{a} \sim 53 \%$ dynamic pressure reduction as soon as the $\mathrm{SW}$ cone angle becomes smaller than $50^{\circ}$. This limit was chosen in order to smooth the swap between radial (reduced dynamic pressure) and non-radial (unchanged dynamic pressure) regimes (Samsonov et al., 2017). In the present study, we employ constant upstream SW conditions. For the sake of covering the full range of cone angles considered in the simulations of (Samsonov et al., 2017), we have performed simulations for the two limit cases of $0^{\circ}$ (referred to as radial IMF) and $\sim 50^{\circ}$ cone angle (referred to as non-radial IMF). We note that there is no consistent usage in the literature of the terms radial or non-radial IMF.

According to Samsonov et al. (2017), the strong total pressure decrease in data seems to be a local, rather than a global, phenomenon. Those authors conceded that their model was not self-consistent in the sense that the modified upstream SW parameter adjustment was global and not specific to the foreshock region for which the statistical results (discussed above) were initially derived. One of the goals of this study is to investigate the role of backscattered ions in producing decreased SW dynamic pressure ahead of the magnetopause by resolving them in a 3D kinetic simulation.

In addition to the expansion of the MP, the other focus of this study is the generation of dawn-dusk asymmetry under radial IMF, which has been investigated for many decades (Akasofu et al., 1982; Akasofu, 1991; Haaland et al., 2017, and references therein). Dawn-dusk asymmetries are ubiquitous features of the coupled solar wind-magnetosphereionosphere system. During the last decades,the increasing availability of satellite and ground-based measurements has made it possible to study these phenomena in more detail (e.g., B. M. Walsh, 2017). Most studies reported so far agree that the dawn-dusk asymmetry is primarily the result of the Parker spiral solar wind impinging with a specific 
geometric configuration that impacts and preconditions the magnetosphere (e.g., Haaland et al., 2017, and references therein). Under radial conditions, the IMF and the SW flow are symmetric with respect to the Sun-Earth line. Under those conditions, one cannot invoke the asymmetry due to the Parker spiral shape to explain any dawn-dusk asymmetry. Therefore, other physical processes, like kinetic effects, should be investigated to explain any potential asymmetry. For instance, statistical studies based on THEMIS and Cluster measurements confirm a rather global expansion of the magnetopause under radial IMF without significant dawn-dusk asymmetries was detected (Zhang et al., 2019). The same statistical study showed that magnetic reconnection (MR) is nearly absent during radial IMF, in contrast to the north IMF conditions during which lobe MR and the consequent dawn-dusk asymmetries are strong (Zhang et al., 2019). However, possible influence of radial IMF on the aurora and dayside magnetic reconnection for different dipole tilt angles have also been investigated by (Østgaard et al., 2003; Zhang et al., 2019).

Kinetic effects are expected to trigger a large set of distinct dawn-dusk asymmetries upstream of the magnetosphere due to the formation of the foreshock region that is connected with solar wind particles backscattered at the bow shock and magnetosheath. Although much of the plasma passes through the bow shock, the reflected population generates a number of plasma instabilities, which trigger waves and generate wave-particle interactions as well as other dynamics at the non-parallel shock that should favor dawn-dusk asymmetries (e.g., B. M. Walsh, 2017, and references therein). The radial IMF condition would thus be the ideal configuration to reveal such kinetic effects and determine their role in producing the dawn-dusk asymmetry so far observed (Zhang et al., 2019). For reference, using Cluster single/multiple spacecraft measurements, Haaland et al. (2014) discussed the dawn-dusk asymmetry at the flanks and at the dayside MP. Similar results were also reported by Haaland et al. (2019), as observed by two of the THEMIS spacecraft, showing the magnetopause being thicker on dawn $\left(\sim 14 \lambda_{i}, \lambda_{i}\right.$ being the ion inertial length) than on dusk $\left(\sim 8 \lambda_{i}\right)$, however no radial IMF conditions were included in the statistical study. Additionally, other observations from INTERBALL-1 and MAGION-4 spacecraft revealed 
asymmetry and deformation at the turbulent magnetopause (Šafránková et al., 2000). Using Geotail observations for northern and southern IMF, Wang et al. (2006) thoroughly discussed the dawn-dusk asymmetry in ion density and temperature based on equatorial distribution of plasma sheet ions.

To interpret the magnetopause motion and the dawn-dusk asymmetry, many sophisticated models have been utilized, ranging from MHD to hybrid simulations. Early theoretical studies showed a contrast of 10\%-20\% between dawn and dusk bulk plasma properties (e.g., Němeček et al., 2002; Walsh et al., 2012), however; those MHD-based models do not explicitly resolve kinetic effects, particularly at the foreshock region. For instance, using a global hybrid model (kinetic ions and fluid electrons), Blanco-Cano et al. (2009) studied radial $\operatorname{IMF}\left(\theta_{v B}=0\right)$ impact on the solar wind interaction with the Earth's magnetosphere. The study focused on the micro-physics processes and wave-particle interactions in the foreshock region but briefly mentioned the dawn-dusk asymmetry issue. Three other models i.e. hybrid, Hall-less and Hall-MHD simulations have been tested in one study by Karimabadi et al. (2004) for the analysis of MR regimes with the conclusion that dawn-dusk asymmetry is obtained and should be related to ions flow. Recently, Turc et al. (2020) used the hybrid -Vlasiator 2D-3V code to study asymmetries in the Earth magnetosheath for different IMF conditions. For reference, the Vlasiator code provides a kinetic description of ions, solving directly the Vlasov equation for the particle distribution function in 2D-3D space, but assumes a fluid description for electrons (e.g., Palmroth et al., 2018). The authors report asymmetries larger than observed for the magnetic field strength, the plasma density, and bulk velocity, a discrepancy that was attributed to using a single set of upstream conditions in their simulations. It is interesting to remark that those authors obtained a stronger asymmetry for magnetic field strength when IMF gets closer to the radial configuration. However, it was not clear how the 2D spatial assumption and the fluid description of electrons in their simulations affected the reported magnetosheath asymmetries. 
Based on the discussion above, two important questions appear: 1) what happens to the magnetopause shape, size, and location if flow-aligned IMF is applied to the system when kinetic effects are included for all species? and 2) does this generate asymmetry in the dawn-dusk and south-north directions so in the dayside magnetosphere?

To answer these questions, we undertake a modeling study utilizing IAPIC, a particlesin-cell code (discussed in section 2). Our strategy is to be able to follow ions and electrons self-consistently with the Maxwell equations describing the fields. Thus the full range of collisionless plasma physics is captured for the macro-ions and macro-electrons involved in IAPIC, yet with limitations due to the grid spatial resolution and assumptions made on the plasma properties (particles density, ion/electron mass ratio, etc.) that we carefully discuss in section 2 (see Blanco-Cano et al., 2006; Eastwood, 2008; Jacobsen et al., 2009; Brackbill, 2011; Masters et al., 2013; Ben-Jaffel \& Ballester, 2014; S. Baraka, 2016). We adopt the initial and the boundary conditions reported in (Suvorova et al., 2010; Samsonov et al., 2017), we used single physical units for each parameter that were used in CCMC reported in (Samsonov et al., 2017).

This paper is structured as follows. This section has introduced the impact of radial IMF orientation on the dynamics of the Earth's magnetosphere and presented a brief survey of observations of asymmetry in planetary magnetospheres. Two IMF orientations, namely, radial IMF $\left(B=B_{x}, 0^{\circ}\right.$ cone angle) and non-radial IMF $\left(|B z|<|B x|<|B y|, \sim 50^{\circ}\right.$ cone angle) will be covered in the current study.

In section 2, we describe the IAPIC code in detail. The scaling of plasma parameters is also presented and tabulated. In section 3, we show our findings regarding the magnetopause motion and the magnetosheath asymmetry, an opportunity to compare to previous modeling results and observations. In section 4 , we discuss in detail the impact of the purely and nonradial IMF on the dynamics of the Earth's magnetosphere, in light of the results obtained so far. In section 5 , we present a summary of our findings and future related work. 


\section{Initial conditions and Simulation Model: IAPIC}

\subsection{Simulation Model: IAPIC}

We use the Institut d'Astrophysique de Paris-Particle-In-Cell EM 3D global code (IAPIC) for treating the plasma kinetically. IAPIC has previously been applied to simulate various magnetospheres in the solar system (S. Baraka \& Ben-Jaffel, 2011; Ben-Jaffel \& Ballester, 2013, 2014; S. Baraka, 2016). IAPIC handles the equations of motion for a large number of macro-particles (macro-ions and macro-electrons) self-consistently under the direct impact of electromagnetic fields through Lorentz force law (S. Baraka \& Ben-Jaffel, 2007; Artemyev \& Zelenyi, 2012).

The code was originally written by (Buneman et al., 1992), which used the boundary conditions reported in (Lindman, 1975) and charge conserving conditions as described in (Villasenor \& Buneman, 1992). We use physical units for each parameter from (Samsonov et al., 2017) and scaled them to IAPIC values using a transformation matrix to convert GSM coordinates to the IAPIC code coordinates (see Fig. 1) as reported in (Cai et al., 2003). The solar wind parameters are tabulated in Table 1 for radial IMF and Table2 for non-radial IMF (see also, Table 1, Cai et al., 2015).

We follow the evolution of the fields and particles within a $61 \times 45 \times 45 R_{E}$ domain with Earth centered and $20 R_{E}$ downstream of the sunward (inlet) side (Table 1). We chose time step $t=3700 \Delta t$ as our comparison point which corresponds to 24 minutes of real time (approximately twice the SW transit time) after starting the simulation. Each time step is equivalent to $\approx 0.38 \mathrm{sec}$ and the spatial resolution of the code is $0.2 R_{E}$. We track $7.0 \times 10^{7}$ particle pairs, with an ion to electron mass ratio of 64 .

Our solar wind parameters for both IMF orientations are given in Tables 1 and 2 for radial and non-radial IMF, respectively. The parameters are set such that a consistent initial conditions are validated before the code run starts, denoted as $t=0 \Delta$, and at the step time, 


\begin{tabular}{|c|c|c|c|c|}
\hline Step time & \multicolumn{2}{|l|}{$t=0 \Delta t$} & \multicolumn{2}{|c|}{$t=3700 \Delta t$} \\
\hline Species/Parameters & ions & electrons & ions & electrons \\
\hline Thermal velocity, $V_{t h i, e}$ & 0.025 & 0.1 & 0.06 & 0.41 \\
\hline Debye length, $\Delta_{i, e}$ & 0.8 & 0.4 & 1.48 & 1.35 \\
\hline Larmor radius, $\lambda_{i, e}$ & 44.95 & 0.7 & 11.51 & 1.31 \\
\hline Gyro-frequency $\omega_{c i, e}$ & $3 . \times 10^{-3}$ & 0.20 & $2 . \times 10^{-3}$ & 0.31 \\
\hline Plasma-frequency $\omega_{p i, e}$ & 0.03 & 0.25 & 0.04 & 0.30 \\
\hline Temperature, $T_{i, e}$ & $1 . \times 10^{-3}$ & $2 . \times 10^{-4}$ & $4.9 \times 10^{-3}$ & $4.09 \times 10^{-3}$ \\
\hline Gyroperiod & 2094.4 & 31.4 & 1289.81 & 20.15 \\
\hline Inertial length $d_{i, e}$ & 16.6 & 2.0 & 13.23 & 1.65 \\
\hline \multicolumn{5}{|c|}{ Unitless values } \\
\hline Step time & $t=0$ & & $t=3700 \Delta$ & \\
\hline Sound speed $C_{s}$ & 0.045 & & 0.0 .1 & \\
\hline Alfvén speed $v_{A}$ & 0.050 & & 0.06 & \\
\hline Alfvén Mach number $M_{A}$ & 2.83 & & 1.4 & \\
\hline Sonic Mach number $M_{s}$ & 3.16 & & 0.92 & \\
\hline Magnetosonic Mach number $M_{m s}$ & 2.0 & & 0.77 & \\
\hline
\end{tabular}

Loaded Simulation Box Information

\begin{tabular}{|l|l|}
\hline grid size & $\Delta=0.2 R_{E}=\Delta x=\Delta y=\Delta z$ \\
\hline Time Step & $\Delta t=\Delta_{x} / \Delta_{v}=7.08$ \\
\hline Simulation box size & $(305 \times 225 \times 255) \Delta$ \\
\hline \# of pair-particles & $7 \times 10^{7}$ ion/electrons pairs \\
\hline Ion to electron mass ratio & 64 \\
\hline Particle density & $n_{i}=n_{e}=4 / \Delta^{3}$ \\
\hline
\end{tabular}

Table 1. Solar wind parameters at the initial state and after $\mathrm{t}=3700 \Delta t$ in the undisturbed solar wind for both ions and electrons for radial IMF. 


\begin{tabular}{|c|c|c|c|c|}
\hline \multirow{2}{*}{$\begin{array}{l}\text { Step time } \\
\text { Species/Parameters }\end{array}$} & \multicolumn{2}{|l|}{$t=0$} & \multicolumn{2}{|l|}{$\bar{t} t=3700 \Delta t$} \\
\hline & ions & electrons & ions & electrons \\
\hline Thermal velocity, $V_{t h i, e}$ & 0.025 & 0.1 & 0.04 & 0.39 \\
\hline Debye length, $\Delta_{i, e}$ & 0.8 & 0.4 & 0.87 & 1.15 \\
\hline Larmor radius, $\lambda_{i, e}$ & 26.26 & 0.4 & 6.85 & 1.12 \\
\hline Gyro-frequency $\omega_{c i, e}$ & $5 . \times 10^{-3}$ & 0.343 & 0.01 & 0.35 \\
\hline Plasma-frequency $\omega_{p i, e}$ & 0.03 & 0.25 & 0.04 & 0.34 \\
\hline Temperature, $T_{i, e}$ & $9 \times 10^{-4}$ & $2 . \times 10^{-4}$ & $2.18 \times 10^{-3}$ & $3.75 \times 10^{-3}$ \\
\hline Gyroperiod & 1256.6 & 18.3 & 1153.15 & 18.02 \\
\hline Inertial length $d_{i, e}$ & 16.1 & 2.0 & 11.71 & 1.46 \\
\hline \multicolumn{5}{|c|}{ Unitless values } \\
\hline Step time & \multicolumn{2}{|l|}{$\Delta t=0$} & \multicolumn{2}{|l|}{$t=3700 \Delta t$} \\
\hline Sound speed $C_{s}$ & \multicolumn{2}{|l|}{0.045} & \multicolumn{2}{|l|}{0.08} \\
\hline Alfvén speed $v_{A}$ & \multicolumn{2}{|l|}{0.05} & \multicolumn{2}{|l|}{0.06} \\
\hline Alfvén Mach number $M_{A}$ & \multicolumn{2}{|l|}{2.83} & \multicolumn{2}{|l|}{1.23} \\
\hline Sonic Mach number $M_{s}$ & \multicolumn{2}{|l|}{3.16} & \multicolumn{2}{|l|}{0.99} \\
\hline Magnetosonic Mach number $M_{m s}$ & \multicolumn{2}{|l|}{2.0} & \multicolumn{2}{|l|}{0.77} \\
\hline \multicolumn{5}{|c|}{ Loaded Simulation Box Information } \\
\hline grid size & \multicolumn{4}{|c|}{$\Delta=0.2 R_{E}=\Delta x=\Delta y=\Delta z$} \\
\hline Time Step & \multicolumn{4}{|c|}{$\Delta t=\Delta_{x} / \Delta_{v}=7.08$} \\
\hline Simulation box size & \multicolumn{4}{|c|}{$(305 \times 225 \times 255) \Delta$} \\
\hline \# of pair-particles & \multicolumn{4}{|c|}{$7 \times 10^{7}$ ion/electrons pairs } \\
\hline Ion to electron mass ratio & \multicolumn{4}{|l|}{64} \\
\hline Particle density & \multicolumn{4}{|c|}{$n_{i}=n_{e}=4 / \Delta^{3}$} \\
\hline
\end{tabular}

Table 2. Solar wind parameters at the initial state and after $\mathrm{t}=3700 \Delta t$ in the undisturbed solar wind for both ions and electrons for non-radial IMF.

\subsection{Initial conditions}

In IAPIC, the spatial and temporal scales (mass ratio and charge to mass ratio, etc ...) are chosen in order to be able to regenerate MHD large-scale classical structure of the Earth's magnetosphere (e.g., Omidi et al., 2004). For their modeling, Samsonov et al. (2017) used MHD and Community Coordinated Modeling Center (CCMC) resources, while the observational data are obtained from ACE, THEMIS and WIND spacecraft. Samsonov 
et al. (2017) studied the impact of non-radial IMF , using a cone angle threshold value of $50^{\circ}$ on the magnetopause size and shape. Contextually, in the current study, we used the physical parameters they used in their MHD initial conditions and scaled them to the IAPIC initial condition values not only for non-radial IMF (where $B_{x}$ and $B_{y}$ are dominant over $B_{z}$, upper limit cone angle case of Samsonov et al. correspond to their threshold value), but also for purely radial IMF (where $B_{y}$ and $B_{z}$ are absent). The purely radial IMF is an additional case included to study differences and similarities with the non-radial nature of IMF on both magnetopause's shape and size and their role in creating dawn-dusk asymmetries. The initial conditions of Samsonov et al. (2017) and our two IMF orientations are then tabulated in Table 3 .

Table 3. MHD initial conditions and their corresponding IAPIC scaled values for radial and non-radial IMF orientation( Samsonov et al., 2017).

\begin{tabular}{|c|c|c|c|}
\hline \multicolumn{2}{|l|}{ Physical Units } & \multicolumn{2}{|c|}{ Code Units } \\
\hline Parameters & MHD & IAPIC $_{\text {radial }}$ & IAPIC $_{\text {non-radial }}$ \\
\hline$N_{S W}(n / c c)$ & 2.606 & 4. & 4. \\
\hline$T_{s w}$ (Kelvin) & 32263 & $5 \times 10^{-4}$ & $5 \times 10^{-4}$ \\
\hline$V_{x}(\mathrm{~km} / \mathrm{s})$ & -470.69 & 0.1412 & 0.1412 \\
\hline$V_{y}(\mathrm{~km} / \mathrm{s})$ & -7.80 & 0 & 0 \\
\hline$V_{z}(\mathrm{~km} / \mathrm{s})$ & -5.09 & 0 & 0 \\
\hline $\mathrm{IMF}_{x}(\mathrm{nT})$ & -2.2 & 0.25 & 0.25 \\
\hline $\mathrm{IMF}_{y}(\mathrm{nT})$ & 2.99 & 0 & -0.34 \\
\hline $\mathrm{IMF}_{z}(\mathrm{nT})$ & 0.659 & 0 & 0.075 \\
\hline Tilt angle & $31^{\circ}$ & $31^{\circ}$ & $31^{\circ}$ \\
\hline Initial Cone $\operatorname{Angle}\left(\theta_{B x}\right)$ & $54^{\circ}$ & $0^{\circ}$ & $54^{\circ}$ \\
\hline Initial Clock Angle $\left(\theta_{C A}\right)$ & $77.6^{\circ}$ & Undefined & $77.6^{\circ}$ \\
\hline
\end{tabular}




\section{Simulation Results}

To our knowledge, a full 3D global kinetic modeling of radial IMF impacts on the dynamics of the magnetosphere has not been published, though the backstreaming of ions in the solar wind flow has been theoretically discussed (e.g., Willis, 1978, Eq. 3). The nonradial IMF event on July, $16^{\text {th }}$, 2007 observed by the THEMIS probes was chosen because it has been the subject of several detailed studies (Jelínek et al., 2010; Suvorova et al., 2010; Samsonov et al., 2017). The solar wind parameters and initial conditions were scaled for IAPIC as described in section 2. For purposes of comparison, we discuss in detail the plasma properties at the time step $3700 \Delta t$ of our simulation for both purely radial and nonradial IMF (IMF orientation corresponding to the threshold cone angle value $\simeq 50^{\circ}$ used by Samsonov et al. (2017) as described in the introduction). This time step corresponds to $\approx 24$ minutes of real time, a relatively long enough period to perform a kinetic simulation of the dayside magnetosphere. In the following, IAPIC simulation results are analyzed to determine the magnetopause shape, size and location for the two IMF conditions considered, which give us a good frame to characterize any dawn-dusk asymmetry in the system.

\subsection{Magnetopause response to radial and non-radial IMF}

We derive the magnetopause's size from the location of the vertical cut of the maximum density gradient steepening using the number density derivative to get the maximum steepening (e.g., Garcia \& Hughes, 2007; J. Lu et al., 2015).

We also use the pressure balance method to derive the MP location, a technique that offers an opportunity to explicitly include kinetic effects. Basically, in the pressure balance method, the dynamic and thermal pressures counterbalance the dipole magnetic pressure. Because of the magnetic field axis tilt $-31^{\circ}$ at the time of THEMIS observation, the system is inherently asymmetric and the Cartesian grid used in the IAPIC simulations is not adequate to accurately derive a density gradient in most planes, particularly in the tilted magnetic equatorial plane. To overcome this difficulty, we transform our Cartesian 3D sim- 
ulation box quantities (density, velocity vector, ... etc, at (x,y,z) positions) into a spherical 3D domain (same quantities at ( $\mathrm{r}, \theta, \phi)$ positions), at the price of losing data from regions outside a spherical volume of radius equal to the smallest dimension of the initial Cartesian box (the Dusk-Dawn and South-North in our case). Our study does not suffer for that limitation because the dayside MP, our region of interest, is located inside the selected spherical domain. After checking that both reference frames provide the same spatial distribution of all physical quantities along the Sun-Earth (OX), the Dusk-Dawn (OY), and South-North (OZ) axis, we focus on deriving the magnetopause size at two key planes, namely the tilted magnetic equatorial plane $\theta=-31^{\circ}$ and the plane $\theta=0^{\circ}$ that contains the Sun-Earth line. To quantify the MP expansion, we inject our initial solar wind conditions in physical units into the Shue model (Shue et al., 1997), which leads to a MP size equals to $10.3 R_{E}$. Therefore, this MP size will be used as the reference in the current study. Our findings are summarized and tabulated in Table 4.

To better visualize asymmetries on large scales of the system, we introduce various contour plots that show a 2D cut of the plasma density distribution and magnetic field topology in 3 planes as shown in Fig. 2 and 3. The dipole field tilt of $\left(-31^{\circ}\right.$, North summer $)$ seemingly has an impact on the macrostructure of the magnetospheric dynamics. Fig. 2ac (d-f) show the 2D contour plots for plasma distribution along the Sun-Earth axis, the Dusk-Dawn axis, and South-North axis for radial IMF (for non-radial IMF).

Fig.2a\&d clearly show the $\left(-31^{\circ}\right)$ tilt angle between the Sun-Earth axis (OX) and the magnetic equator axis for radial and non-radial IMF, respectively. Therefore, the forefront of the solar wind coplanar inflow approaches the planet and hits the upper boundary of the magnetosphere before it comes in head-on combat with the magnetic equator axis. This results in squeezing the magnetopause at high latitude and relaxes it in lower latitude. This torque-like effect generates plasma streaming that has a flare out configuration for radial IMF and a confined configuration for non-radial IMF as in Fig. 2d. For both IMF orientations, localized density maximas (yellow areas) can be seen at the subsolar point 
(stagnation region) close to OX although being extended southward for the non-radial case. These regions extend azimuthally duskward for both IMF orientations (Fig. 2b\&e). Also, large denser regions are present near the north cusp for both orientations although a smaller one can be seen near the south cusp too for the non-radial case. In the linear plot Fig. 8, it is seen like a hump at $\approx-16 R_{E}$ along the Sun-Earth line for non-radial IMF. We do not know why this layer appears for non-radial but not for radial IMF. The relaxation of the southern part of the magnetosphere showed a denser plasma population up to $30 R_{E}$ tailward and flared in toward north at around $25 R_{E}$ for non-radial IMF in Fig. 2 d. The cavity around the planet position is smaller and more confined in the non-radial IMF case than in the radial IMF case. Moreover, Fig. 2b\&e show the equatorial plane plasma distribution for radial and non-radial IMF, respectively. Particle entry inside the magnetosphere is largely distributed around the planet making the cavity reaches $\pm 5 R_{E}$ along the Dusk-Dawn direction and around $3 R_{E}$ tailward, with plasma tube along the Sun-Earth line up the planet position. On the other hand, the effect of $B_{y}$ for the non-radial IMF in Fig. 2e, shows that the magnetopause is compressed on both dusk and dawn sides. The cavity around the planet is more confined and reduced in size to $\pm 5 R_{E}$ along South-North direction for radial IMF, and $\pm 4 R_{E}$ for non-radial IMF, respectively (Fig. 2c\&f). The plasma density in the SouthNorth/the Dusk-Dawn plane for radial IMF (Fig. 2c) shows denser plasma in the dusk sector from -10 to -20 $R_{E}$ than in the dawn sector from 10 to $20 R_{E}$, while in the northern sector there is a denser plasma that extends from around 10 to $17 R_{E}$ but not regularly structured with same thickness in the southern sector. It appears that there is a finger-like structure (particle entry, small blue region) at around $-5 R_{E}$ on the duskside that has an extension of about $1 R_{E}$ in the cavity around the planet. On the other hand, for non-radial IMF the plasma distribution contour shows smaller cavity size and also a denser plasma on the dusk side(Fig. 2f).

Fig. 3 shows the corresponding magnetic field lines topology of plasma density contour shown in Fig. 2, respectively. This figure sheds light on the differences and similarities between two IMF orientations along three different planes. 
In Fig. 3a, the radial IMF field lines along OX are horizontal at $-20 R_{E}$ and $\pm 3 R_{E}$ along South-North direction and seen curled at $\pm 10 R_{E}$. At the magnetopause position, the field lines divert at $\mathrm{f}(\mathrm{x}, \mathrm{z})=(-10,-8) R_{E}$. On the dayside magnetosphere, there are two potential lobe magnetic reconnection sites(MR) sites. They are approximately located in the southern hemisphere at $\mathrm{f}(\mathrm{x}, \mathrm{z})=(0.5,-12)$ and at $(-7.6,11.9) R_{E}$ in the northern hemisphere. The magnetic field line topology shown in Fig. 3d is horizontal in the undisturbed SW; this was not the case in Fig. 3a. This difference is attributed to the impact of $B_{y}$. In Fig. 3d potential lobe MR sites are seen also at $f(x, z)=(-8 .,-12$.$) in the southern hemisphere and$ at $(5,15) R_{E}$ in the northern hemisphere. Constant attention should be made when looking at Fig. 3b, taken in the equatorial plane, because of the dipole tilt, what is shown here for radial IMF is the high latitude magnetopause along OX in the Dusk-Dawn direction. It is found that field lines from IMF connect to dipole field and permit particle entries at that latitudes. The wavy structure in the night-side (not the focus of the current study) indicates a complex current system induced at that distance. A potential MR site is shown at $\mathrm{f}(\mathrm{x}, \mathrm{y})=(-7.6,9.9) R_{E}$. The curling of magnetic field lines at $\mathrm{f}(\mathrm{x}, \mathrm{y})=(5,-15),(-15,-7) R_{E}$ corresponds to the plasma dynamics shown in Fig. 2d. Same in Fig. 2e for non-radial IMF, the curled magnetic field lines at a latitude corresponding to $\approx 6 R_{E}$ (north) are directed toward dusk-midnight direction. Potential MR sites are at $\mathrm{f}(\mathrm{x}, \mathrm{y})=(4.4,5.9),(-8.6,0.1),(3.5,-$ 7.9) $R_{E}$. In Fig. 3c, the magnetic field topology shows a more extended structure of closed magnetic field lines for both duskside and dawnside than the non-radial case. The regions extends until $\approx \pm 14 R_{E}$ along the dusk-dawn axis and reach up $\pm 12 R_{E}$ along the southnorth axis. In contrary, Fig. 3f shows different structures, where the extension of field lines is less important along the dusk-dawn axis due to large scale connections of planetary and interplanetary magnetic field lines and clear MR sites located in the southern hemisphere on both dusk and dawn sides at $\mathrm{f}(\mathrm{y}, \mathrm{z})=(-12.5,-2.6),(14 .,-3.) R_{E}$, respectively. Such reconnection sites are not present (at least in this plane) for the radial case. The detailed investigation of the MR regions is out of the scope of the present study yet these results suggest that these reconnection regions are formed in the northern and southern lobe regions and extend to 
the dawn and dusk flanks. They could lead to the squeezing of the dusk and dawn flanks of the magnetopause seen in Fig. 2e.

In the first step, we focus on the direction defined by $\phi=-180^{\circ}$ in both planes. Using the maximum density steepening technique, we derive comparable values for the magnetopause position at $(\sim 10.6, \sim 11.0) R_{E}$, respectively for radial and non-radial IMF along the Sun-Earth axis. We also obtain the same size when the effect of backstreaming ions is removed (by post-processing) from the bulk flow for both IMF orientations. It would suggest as it will be confirmed below that while the contribution of the backstreaming ion population to the total density is small and does not change the location of the density gradient maximum their contribution to the total pressure is important. In particular, finite Larmor radius effects are more important for energetic ions. Along the tilted magnetic equatorial axis, the magnetopause positions are $(11.6,12.0) R_{E}$ with bulk flow, which also remain unchanged without backstreaming ions, respectively for the two IMF conditions.

First, we note that the different magnetopause positions derived from the IAPIC simulation are all larger than the expected magnetopause position $\left(\sim 10.3 R_{E}\right)$ derived from the Shue model (Shue et al., 1997). We remind that this reference MP size corresponds to the initial solar wind physical parameters used in our simulations. All values derived show a sunward expansion of the magnetopause position along the two selected axes. The magnetopause appears to be subjected to a reduced SW dynamic pressure that allows the dipole magnetic field network to expand outward. Based on the maximum density steepening technique, it is remarkable that our model predicts the magnetopause expansion self-consistently in the range (0.3-1.3) $R_{E}$ along the Sun-Earth axis and tilted magnetic equatorial axis for the radial IMF case but also (0.7-1.7) for the non-radial IMF, respectively.

In the following, we explore our 3D IAPIC simulation results to try to uncover potential processes that could be at the origin of the measured expansion. Since early reports, several studies pointed to the potential impact of kinetic effects, particularly with the detection of the signature of particles streaming in a direction opposite to the solar wind (Spreiter \& 
Alksne, 1969; Willis, 1978; Sibeck et al., 2001; Samsonov et al., 2017). As IAPIC simulations offer the access to all populations of particles (macro-particles) with specific kinetic properties, we tried to extract those particles on the dayside that move sunward, against the main impinging solar flow. That statistical sub-population of particles has its own kinetic properties and most importantly counterbalances the ram pressure of the incident solar flow, as if it was originating from the magnetosphere and flowing outward. It is important to stress that this population has kinetic properties (temperature, speed, etc.) much different from the planetary ionospheric population that flows from the plasmasphere or the polar wind, In Fig. 4, bulk pressures (dynamic, thermal denoted $P_{d y n}$ and $P_{t h m}$, respectively) are shown with and without taking into account the contribution of backstreaming ions to highlight the difference they make in the pressure balance. First, we remark that the thermal pressure is dominant over dynamic pressure in the magnetosheath as expected for a conversion of the convective flow energy to thermal energy after the bow-shock crossing. $P_{d y n}$ and $P_{t h m}$ cross $P_{m a g}$ at two points, i.e. with and without backstreaming ions included. The corresponding size of the MP is $\sim 10.8 R_{E}$ for radial IMF and $\sim 11.7 R_{E}$ for non-radial IMF along the Sun-Earth axis. Importantly, if the backstreaming ion effect is removed, then the magnetopause size drops down to 10.5 and $11.4 R_{E}$ for the same IMF orientations, respectively. The magnetopause is also measured along the tilted magnetic equatorial axis with and without backstreaming ions and found equal to $11.9,11.5 R_{E}$ for radial IMF and $12.0,11.6 R_{E}$ for non-radial IMF, respectively. To summarize, these findings are tabulated in Table 4. It is worth noting that the expansion ranges when using the pressure balance method for deriving the MP size is slightly different from that using the density steepening method. For example, the MP expansion rate is $0.5-1.4 R_{E}$ for radial and non-radial IMF along the Sun-Earth axis, compared to $0.3-0.7 R_{E}$ derived by maximum density steepening method. The difference between the two measurements is $0.2-0.7 R_{E}$. The expansion range is $1.6-1.7 R_{E}$ as derived by pressure method compared to $1.3-1.7 R_{E}$ as measured by density steepening method, respectively along tilted magnetic equatorial axis. With only a difference between the two methods of $0.3 R_{E}$ for radial IMF and similar values for non-radial IMF 
along the tilted magnetic axis. Surprisingly, both methods suggest that the magnetopause expansion at the subsolar point is larger for the non-radial case than for the radial case. It is probably the effect of the diffuse backstreaming ions in the case of non-radial IMF which are hotter than that of radial IMF which explains the expansion toward the sun as well, as a reference is the cross-field diffusion discussed in (Tsurutani \& Lakhina, 1997; Paschmann et al., 1981).We emphasize that the ring current that generates the dipole field for both radial and non-Radial IMF is the same. That's to say, the solar wind conditions, and the dipole strength are the same for both IMF cases, except for the non-radial IMF, where $B_{y}$ is dominant over $B_{x}$ and $B_{z}$. Therefore, any noticeable differences such as the larger MP expansion is directly related to the presence of the IMF $B_{y}$. It is worth to continue broadly investigating the radial IMF by PIC code to better understand the related physics that handle this problem.

\section{Maximum Density Steepening magnetopause derivation}

\begin{tabular}{llllll} 
IMF / Axis & \multicolumn{2}{l}{ The Sun-Earth Axis } & & \multicolumn{2}{l}{ Tilted Magnetic eq } \\
\cline { 2 - 3 } Kinetic effect & Yes & No & Yes & No \\
magnetopause for radial IMF & $10.6 R_{E}$ & $10.6 R_{E}$ & $11.6 R_{E}$ & $11.6 R_{E}$ \\
magnetopause for non-radial & $11.0 R_{E}$ & $11.0 R_{E}$ & $12.0 R_{E}$ & $12.0 R_{E}$
\end{tabular}

\section{Pressure balance magnetopause derivation}

\begin{tabular}{llllll} 
IMF/Axis & \multicolumn{2}{l}{ The Sun-Earth Axis } & & \multicolumn{2}{l}{ Tilted Magnetic equatry } \\
\cline { 3 - 5 } Kinetic effects & Yes & No & Yes & No \\
magnetopause for radial IMF & $10.8 R_{E}$ & $10.5 R_{E}$ & $11.9 R_{E}$ & $11.5 R_{E}$ \\
magnetopause for non-radial & $11.7 R_{E}$ & $11.3 R_{E}$ & $12 . R_{E}$ & $11.6 R_{E}$
\end{tabular}

Table 4. Summary of results for the MP location is tabulated as derived by density steepening method (derivative of number density) and pressure balance methods for both IMF orientations. The values are measured on both the Sun-Earth Axis and tilted magnetic equatorial axis. The kinetic effect is when the bulk flow (including backstreaming ions) is considered, and its absence when inflow (earthward) only is considered. The reference size for these values is $10.3 R_{E}$. 
In their papers, Bonifazi and Moreno (1981b, 1981a) used ISEE2 data of 3253 ion spectra that correspond to $\approx 90$ hours, studying the density and energy of the backstreaming ions. They quantitatively characterized them into three categories. The authors used a scaling factor such that the ratio $(A)$ of the backstreaming bulk speed over their thermal speed to identify the following three populations: $\geq 1.2$ for reflected ions, $\leq 0.8$ for diffuse ions and an intermediate population $0.8 \leq A \leq 1.2$.

Here, we adopted their method to quantify the characteristics of the backstreaming ions in the dayside solar wind flow. First, a simple statistical study of the dayside populations shows that the solar wind bulk flow is composed by $16.5 \%$ and $20 \%$ of backstreaming ions for radial and non-radial IMF, which confirms that their contribution to the total density is small whereas its contribution to the total pressure can be crucial. Fig. 5a shows that, for radial IMF $72.5 \%$ of backstreaming ions are characterized as reflected ions and only $27.5 \%$ as intermediate $(0.8<A<1.2)$. For the non-radial IMF case, we found a totally different characteristics for backstreaming ions. There are only identified as $21.6 \%$ reflected particles, $31.4 \%$ intermediate particles and $47.0 \%$ diffuse particles along the same OX axis. To follow up the impact of the tilted magnetic axis on the particles populations, we also conducted the same statistical analysis on the tilted magnetic equatorial axis. We found for radial IMF that $59 \%$ of ions are reflected and $41 \%$ are in the intermediate phase. On the other hand, backstreaming ions are characterized as $23.5 \%$ of reflected ions, $53 \%$ of intermediate case, and finally $23.5 \%$ for diffuse ions for non-radial IMF. In Fig. 5 we found that for radial IMF, on both axis, most of the backstreaming ions are in the reflection mode, with a small percentage in intermediate mode at the bow shock. On the other hand, for non-radial IMF, on both axis, backstreaming ions are in intermediate and diffuse mode in the solar wind, but the reflection mode appears to be dominant around the bow shock.

Still, to better understand the kinetics of the distribution of the backstreaming ions, we show in Fig. 6 the velocity distribution function (VDF) of solar wind ions along the Sun-Earth line as far as $-20 R_{E}$. For the purely radial IMF case, a substantial fraction 
of VDF spatial distribution of backstreaming ions is found in the three planes (XZ, XY and YZ) whereas only a small fraction is obtained for non-radial IMF, especially in the YZ plane. The VDF in Fig. 6a shows the reflection of the plasma sunward and southward for radial IMF, with minor reflection for non-radial IMF but larger distribution toward north and earth direction as confirmed by Fig. 5. It is also found that at $\mathrm{Y}=\mathrm{Z}=0$ and $\mathrm{X}=-20 R_{E}$ that the reflection of backstreaming ions are larger for radial than non-radial IMF. While on the other hand in Fig. 6c\&f, the spatial distribution of ions is reflected mostly toward the south and sunward for radial and mostly toward north for non-radial IMF.

In the following, we track the magnetopause shape for both IMF orientations in two different locations. In order to handle the complex geometry of the tilted dipole, we use spherical coordinates such that $\phi=-180^{\circ}$ at the dayside standoff distance $(\theta=0)$ along the Sun-Earth Axis and $(\theta=-31)$ along the tilted magnetic equatorial axis. In that frame, we track the shape every $20^{\circ}$ along $\phi$ in two different axes. The MP shape for the two IMF orientations is constructed in the XY-plane and in the magnetic equator that both contain the dusk-dawn direction. In Fig. 7, the MP is shown in the half-plane that contains dawn (positive Y), so that the asymmetry between dusk and dawn clearly appears using a mirror image of the MP shape in the dusk half-plane that is over-plotted on the dawn half-plane. For example for the radial case, Fig. 7a\&b show a small the dusk-dawn asymmetry for the two selected planes (i.e. XY and tilted magnetic equator planes) whereas this asymmetry is more important in the non-radial case; the duskside magnetopause being more extended than the dawnside (Fig. 7c\&d).

In particular Fig. 7c, the MP along the Sun-Earth axis is squeezed earthward on the dawnside down to $7.5 R_{E}$ whereas the dusk sector spreads out to $\approx 12 R_{E}$. In Fig. $7 \mathrm{~d}$, we have a peculiar non-symmetric MP shape in the magnetic equator with a size $\approx 10 R_{E}$ except near the Y-axis. In Fig. 8, the linear densities for both IMF orientations are plotted in three different directions. For purely radial IMF (Fig. 8a\&b, ion and electron densities are over-plotted along the Sun-Earth line (upper panel $\mathrm{OX}, \mathrm{Y}=\mathrm{Z}=0$ ) from -20 to $-10 R_{E}$, the 
Dusk-Dawn line (middle panel OY, $\mathrm{X}=-13, \mathrm{Z}=0$ ) from -20 to +20 , and South-North line (lower panel OZ, $\mathrm{X}=-13, \mathrm{Y}=0$ ) measured. Densities in $\mathrm{OY}$, and $\mathrm{OZ}$ panels are aimed to show the density profile just outside the MP position taken at $-13 R_{E}$. The vertical dashed line in the upper panel shows where the densities are plotted in the other two directions. Fig. 8 shows the quasi-neutrality of the solar wind, magnetosheath and magnetospheric plasmas. Ion and electron densities are correlated with no charge separation. Also, it is found that along the Dusk-Dawn axis and South-North axis taken just outside the MP there is an apparent asymmetry at both the dusk-dawn and south-north directions. For both IMF orientations, magnetosheath densities are larger on the duskside than on the dawnside and larger in the southern hemisphere than in the Northern.

For the non-radial case (Fig. 8b), we notice a hump/heap just upstream of the bow shock position at $\approx-16 R_{E}$. We tracked backstreaming ions at that distance and only a little fraction was found. Its type corresponds to reflected ions according to the characterization of backstreaming ions reported in Fig. 5. The plasma jump at $\mathrm{X}=-16 R_{E}$ reaches factor 2 . The same value is found when we perform a cut along $\mathrm{OY}$ and $\mathrm{OZ}$ at $\mathrm{X}=-16 R_{E}$ (not shown) indicating that this jump is at a large scale and present across the full magnetosheath. The other major components of the solar wind dynamics is related to the ion velocity modulus that is shown in Fig. 9 in the same order. The density hump in Fig. $8 \mathrm{~b}$ corresponds to a velocity decrease in Fig. 9b.

The temperatures of solar wind particle species are important to identify boundary crossing, for instance, between the cold component of the solar wind and the hotter component of the magnetosheath plasma. Furthermore, ion-to-electron temperature $T_{i} / T_{e}$ is approximately conserved at the MP boundary (Wing et al., 2014). The $T_{i} / T_{e}$ ratio in the magnetosheath was thoroughly investigated by Lavraud et al. (2009). In the current paper, the $\left(T_{i} / T_{e}\right)$ ratio of the impinging solar wind is depicted in Fig. 10. For radial IMF Fig. 10a, $T_{i}$ is slightly fluctuating in the solar wind until it increases by a factor 2 between -15 to $-12 R_{E}$ along OX as expected due to the shock crossing which slows down and heats the 
plasma; then it decreases downstream before it increases again in the magnetosheath until it reaches the MP position. While on the other hand, the electron temperature $T_{e}$ in the magnetosheath maintains a constant value and has increased by a factor 1.2 compared with the electron temperature in the solar wind. The average $\left(T_{i} / T_{e}\right)$ ratio in the magnetosheath is $\approx 1.72$. The temperature profile for the non-radial IMF case shown in Fig. 10b is different from the purely radial IMF case. $T_{i}$ starts constantly increasing at $-15 R_{e}$ including the bow shock and the magnetosheath. From the solar wind to the magnetosheath, $T_{i}$ increases by a factor 4 . On the other hand, $T_{e}$ starts increasing at $-16 R_{E}$ and keeps rising through the magnetosheath. The average $\left(T_{i} / T_{e}\right)$ ratio in the magnetosheath is $\approx 1.1$. The ion-to-electron temperature ratio is discussed more in detail in section 4

The temperature anisotropy was studied extensively in the past (Gingell et al., 2015; Karimabadi et al., 2014; Treumann \& Baumjohann, 2013). In the current paper, we were able to derive the temperature anisotropy of the solar wind particle species. Fig.10c shows for radial IMF, the average ion temperature anisotropy $T_{\perp i} / T_{\| i}$. In the dayside sector (between -18 and $-10 R_{E}$ ) this ratio is $\approx 2$. and inside the magnetosheath (between -14 and $\left.-12.5 R_{E}\right)$ it reaches 2.7. Therefore the perpendicular ion temperature anisotropy is stronger in the magnetosheath than in the solar wind. The maximum ratio is found at the bow shock $\left(\sim-14 R_{E}\right)$ with a factor of 3.3. The average electron temperature anisotropy $T_{\perp e} / T_{\| e}$ in the dayside sector equals to $\approx 2.3$, which is almost the same as inside the magnetosheath. Fig. 10d displays for the non-radial IMF orientation, the anisotropy average for ions in the dayside sector (between -18 and $-10 R_{E}$ along $\mathrm{OX}$ ). The $T_{\perp i} / T_{\| i}$ ratio $\approx 6$ for ions and $\approx 1.8$ for electrons. In the magnetosheath (between -14 and $-12.5 R_{E}$ along OX) $T_{\perp i} / T_{\| i}$ ratio is 3.2 whereas electron temperature is quite isotropic. These results will be discussed in section 4 . 


\subsection{Dawn-Dusk asymmetry in the dayside magnetosphere under the influ- ence of radial and non-radial IMF}

The large scale results reported above all indicate systematic asymmetries in the DuskDawn and South-North directions for the two IMF orientations. In the following, we try to quantify those asymmetries, particularly in the Dusk-Dawn direction for the radial and non-radial IMF cases we have simulated. Figures $2 \mathrm{~b}, \mathrm{c}, \mathrm{e}, \mathrm{f}, 7$, and 8 visually show asymmetry in the Dusk-Dawn direction.

Fig. 2b,e show the Dusk-Dawn asymmetry along the Sun-Earth direction (OX) and Fig. 2c,f show the asymmetry along the Dusk-Dawn (OY) and South-North (OZ) directions. The linear density plots are shown in Fig. 8, middle panel, plotted along the dusk-dawn direction at $X \approx-13 R_{E}$. The asymmetry in the South-North planes can be seen in Fig. 8 lower panel. In Fig. 11, some solar wind parameters i.e. $N_{i}, V_{i}$, and $T_{i}$, and Fig. $12 B_{x}$, $B_{y}$ and $B_{z}$ are plotted along three different axes (parallel to OX) for each IMF orientation. Two of which are plotted at $Y= \pm 6 R_{E}$ on both sides of OX axis, and the third along the Sun-Earth line from -18 to 10.8 and $11.7 R_{E}$ for radial and non-radial IMF. Dashed lines are the calculated positions of the MP at dawn and dusk $\left(Y= \pm 6 R_{E}\right.$, green and red dashed lines, respectively) and along $\mathrm{OX}(Y=0$, blue dashed line) as measured from the gradient density method (same as shown in Fig. 7).

In the magnetosheath, left to the vertical blue dashed line, all plasma parameters are asymmetric. On the dusk side (red dashed line), the MP position is located at -8.5 , and $-7.7 R_{E}$ for radial and non-radial IMF. Whilst on the dawn side (green dashed line), it is located at -7 , and $-5.2 R_{E}$. For both IMF orientations, ion density increase in the magnetosheath is larger on the duskside than on the dawnside (Fig.11). The largest increase is along the symmetry axis (OX). For the radial case, ion velocity $\left(V_{i}\right)$ decreases after the bow shock crossing along (OX) whereas this deceleration is less important for both dawn and dusk sides until reaching the magnetopause. For the non-radial case, the same behavior is shown along (OX) whereas the ion flow seems to accelerate until the magnetopause crossing on the 
duskside and to decelerate on the dawnside even more than along (OX). Ion temperature $T_{i}$ in the solar wind and in the magnetosheath is larger on the dawnside for the radial case whereas it does not show any the dusk-dawn asymmetry for the non-radial case. In Fig. 12, for both IMF orientations, $B_{z}$ becomes positive close to the magnetopause likely due to the draping effect. Furthermore, the large initial IMF $B_{y}$ of non-radial case (positive in GSE, negative in IAPIC coordinates=-0.34) remains quite constant and homogeneous in the solar wind and magnetosheath regions. For the radial case, the By component is more variable and different between dusk and dawn side in the solar wind and magnetosheath regions. Initial IMF $B_{x}$ (negative in GSE and positive in IAPIC coordinates $=0.25$ ) does not remain constant and shows the Dusk-Dawn asymmetry. A large increase occurs only in the dawn magnetosheath sector. For the non-radial case, Bx remains quite steady in the dayside region but is larger on the duskside than along OX and on the dawnside where the initial value has been reduced.

\section{Discussion and Analysis}

As described in section 3.1, we derived the MP position $(10.6,11.0) R_{E}$ along the SunEarth line for purely and non-radial IMF, respectively (using the location of the maximum number density gradient). In addition, we have shown that PIC simulations offer the possibility to isolate backstreaming ions from the pool of particles in the box simulation. This process allows us to derive their contribution to the dynamic and thermal pressures in the dayside magnetosphere. It is important to stress that other complex effects could be induced by the presence of those backstreaming particles, like induced currents and fields, that will be considered in a future study. Subtracting the contribution of backstreaming ions in the ensemble average of the plasma properties increases the resulting system pressure and consequently moves the magnetopause location toward Earth.

Using the balance between ram pressure and magnetic pressure (e.g., Willis, 1978, Eq. 3) we were able to derive the MP size in different planes with and without backstreaming 
ions (Table 4). These calculations reveal the impact of backstreaming ions in reducing the solar wind dynamic pressure allowing for MP expansion by $0.3-0.4 R_{E}$. All our findings above also suggest the important results that by neglecting ions kinetic effects, MHD simulations implicitly overestimate SW pressure in the dayside region and underestimate the MP size.

For instance, the MP expansion range derived here is consistent with the expansion observed by THEMIS spacecraft for the event of 16 July 2007. To get the observed expansion with their MHD model, Samsonov et al. (2017) were forced to modify the solar wind properties, propagated from WIND and ACE in situ measurements, of their boundary conditions. This means that a non-negligible fraction (up to $\sim 40 \%$ ) of the observed expansion that is due to backstreaming particles was modeled in their study by an ad hoc reduction of the SW dynamic pressure.

We conclude that the dynamic pressure of backstreaming ions contributes significantly to the expansion/compression of the magnetopause. Note that when the magnetopause is derived from the density maximum steepening, the effect of the backstreaming ions is not significant as their contribution to the total density is small. This indicates that the main driver of the expansion of the magnetopause is the upstream reduction of the solar wind dynamic pressure in the dayside region due to the reduction of the solar wind bulk flow by the presence of the backstreaming ions, which although being a small percentage of the total solar wind ion population ( $16.5 \%$ and $20 \%$ for radial and non-radial IMF, respectively) have a strong effect on the dynamic pressure. More generally, the insensitivity of the MP size to the density of backstreaming ions confirms the conclusions reported in (Samsonov et al., 2020, Eq. 2), that density and velocity (dynamic pressure) might have different contributions to the effective values of the dynamic pressure component in the pressure system balance used in driving the magnetopause position. This appears clearly in our results in Table 4.

However one can notice that for the non-radial case with a large positive IMF $B_{y}$ component, the effect of backstreaming ions could have been expected to be more important on the dawnside where the foreshock region is moved leading to a pronounced magnetopause 
expansion on the same side. Instead, we found that the magnetopause is moved earthward in both dawn and dusk sectors. We suspect that this effect could be due to the formation of magnetic reconnection regions across the dayside magnetopause seen in Fig. 3 in these specific IMF conditions (with a clock angle of $77.6^{\circ}$, a cone angle of $54^{\circ}$ and a large dipole tilt of $-31^{\circ}$ corresponding to a North summer time) rarely investigated in past studies (e.g., Trattner et al., 2007). Indeed, the dayside reconnection process is known to erode the magnetopause therefore moving earthward the magnetopause boundary. Our results could suggest that the reconnection process which erodes the magnetosphere could dominate against the effect of the backstreaming ions which tend to expand the magnetopause. The detailed investigation of the magnetic reconnection process in our simulations is out of the scope of the present study and will be addressed in a further study.

Using THEMIS measurements, Wang et al. (2012) showed how the $T_{i} / T_{e}$ ratio changes in the magnetosheath and the plasma sheet. They showed that $T_{i} / T_{e} \approx 4-12$ in the magnetosheath. In the tail plasma shee, this ratio spans $\approx 6-10$. Other studies statistically examined the $T_{i} / T_{e}$ in different regions in the magnetosphere to find a ratio $\approx 6$-12 close to the magnetopause (Lavraud et al., 2009, and references therein) . On the other hand the $T_{i} / T_{e}$ ratio was as low as $\approx 3$ at the magnetosheath flanks (Lavraud et al., 2009). In the current paper, we show in Fig. 10a\&b, the ion and electron temperature ratio $T_{i} / T_{e}$ in the dayside region. In the magnetosheath this ratio is found about 1.72 and 1.1 for radial and non-radial IMF, respectively (Dimmock et al., 2014, 2015).

On the other hand, the presence of backstreaming particles should also affect the temperature anisotropy in the dayside region ahead of the magnetosphere. Grygorov et al. (2017); Samsonov et al. (2012) showed that the temperature anisotropy in the magnetosheath controls the pressure distribution near the magnetopause. Here we found that the ion temperature anisotropy ratio is about 2 in the magnetosheath for radial IMF, and about 6 for non-radial IMF (Fig.10c\&d), consistent with Wang et al. (2012). Analysis of 
the temperature distributions will be used in future work to study microinstabilities and reconnection.

To characterize the kinetic properties of the backstreaming particles, we have first used the nomenclature of Bonifazi and Moreno (1981b) and obtained results given in section 3. In Table 5, we show the bulk speed of backstreaming ions in real physical units in accordance with the characterization factor reported in (Bonifazi \& Moreno, 1981b). It is found that for both IMF orientations and along both axes, the magnitude of the bulk velocity of the backstreaming ions (directed sunward) is larger than the full solar wind velocity. Despite a relatively small percentage of the total ion population, backstreaming ions reduce significantly the solar wind velocity. Furthermore, thermal velocities of backstreaming ions are larger for the non-radial case than for the radial case, suggesting that heating of backstreaming ion is more important in the non-radial case.

The second major finding of this study is the Dawn-Dusk asymmetry. Uncovering its origin is important for better understanding, modeling and prediction of space weather phenomena (e. g., S. Lu et al., 2016). Asymmetry is observed by Cluster spacecraft in north-south magnetotail planes (Haaland et al., 2017; Samsonov, 2006) and in dawn-dusk planes (A. P. Walsh et al., 2014; Samsonov, 2011; Dimmock et al., 2017; Turc et al., 2020). Both observations and numerical simulations have revealed that the magnetopause size is a function of IMF strength and orientation, and solar wind dynamic pressure, which by turn modify the magnetopause shape and generate dawn-dusk asymmetries (Liu et al., 2019). Using data from IMP 8 and ISEE1, ISEE 3 and WIND, (e.g., Paularena et al., 2001, Table 3) showed a significant dawn-dusk asymmetry in the Earth's magnetosheath which is larger on the dawn side than on the dusk side, for all IMF orientation. They also showed that the IMF orientation impacts density asymmetry in dawn-dusk direction. Paularena et al. (2001) reported the same kind of asymmetry in different regions in the dayside magnetosphere in the Sun-Earth and the Dusk-Dawn plane. In their recent study, Turc et al. (2020) discussed the magnetosheath asymmetry in terms of IMF, solar wind 
Total number of particles inside the simulation box

\begin{tabular}{|l|l|l|}
\hline & \multicolumn{1}{|c|}{ Radial } & \multicolumn{1}{c|}{ non-radial } \\
\hline \# bulk flow ions & $5.38958 \times 10^{7}$ & $5.21372 \times 10^{7}$ \\
\# backstreaming ions & $8.87744 \times 10^{6}$ & $1.07256 \times 10^{7}$ \\
percentage & $16.5 \%$ & $20 \%$ \\
\hline
\end{tabular}

Backstreaming Ions Characteristics

\begin{tabular}{|l|l|l|l|l|}
\hline & \multicolumn{2}{|c|}{ Radial } & \multicolumn{2}{c|}{ non-radial } \\
\hline & OX & Tilt & OX & Tilt \\
\hline reflected ions & $72 . \%$ & $58 . \%$ & $22 . \%$ & $24 . \%$ \\
intermediate ions & $27 . \%$ & $41 . \%$ & $31 . \%$ & $53 . \%$ \\
diffuse ions & $1 . \%$ & $1 . \%$ & $47 . \%$ & $23 . \%$ \\
$V_{b p}\left(k m . s^{-1}\right)$ & 373 & 392 & 294 & 346 \\
$V_{\text {thm }}\left(k m . s^{-1}\right)$ & 254 & 255 & 321 & 332 \\
$V_{s w}\left(k m . s^{-1}\right)$ & 287 & 254 & 233 & 248 \\
\hline
\end{tabular}

Table 5. The ratio of backstreaming ions to bulk flow of the number density for both IMF orientations is shown in the upper panel. The backstreaming ions are characterized with three categories (see Fig. 5). Based on scaling factor ((Bonifazi et al., 1980)) the backstreaming ions are reflected, intermediate or diffuse. $V_{b p}$ is the bulk flow of the backstreaming ions, $V_{t h m}$, their thermal speed and their full solar wind velocity $V_{s w}$ (including contribution of backstreaming ions) are shown in the lower panel for both IMF orientations along OX and Tilt axis. This data is measured at the dayside magnetosphere

This article is protected by copyright. All rights reserved. 
density, velocity by using Vlasiator hybrid code (Palmroth et al., 2018). They found that magnetic field asymmetry and density variability in the magnetosheath are stronger when IMF tends toward a radial direction.Similarly, using IAPIC, the dawn-dusk asymmetry in the magnetosheath and in the solar wind is investigated. In our work, the MP shape in Fig. 7, shows how asymmetric the dawn-dusk sides are. A result that is confirmed in Fig. 11, and 12 , IMF, density, temperature and ion velocity are tested at the the dusk-dawn flanks asymmetry in the magnetosheath. In the magnetosheath (left to the vertical blue dashed line) all plasma parameters are asymmetric which is consistent with recent results obtained by Turc et al. (2020). Furthermore, for both IMF orientations, the magnetopause is more extended on the duskside (red dashed line) than on the dawnside (green dashed line).

Finally, our analysis of the location, shape and size of the MP with the techniques developed for that purpose, in addition to the ability to quantify plasma parameters in 3D to track asymmetries in the dusk-dawn and south-north direction, our code is applicable to planetary and exoplanetary magnetospheres. Furthermore, our findings can also contribute to alternative methods for a better analysis of soft x-ray imaging of the magnetosphere (Sibeck et al., 2018) in a complementary manner. This includes the MP, the cusp dynamics, the magnetosheath that is related to density structure which can be deduced from soft x-ray observation.

Most current support to the SMILE mission is based on MHD modeling (SMILE working group). In light of the results obtained so far (see Figs. 4 and 7 , and Tables 4), our global 3D electromagnetic kinetic code provides another point of view on the range of expected boundary locations under various solar wind flux. An accurate estimation of those boundary locations are key to interpret the X-ray signal that will be detected by SXI, the SMILE X-ray detector. In addition, our simulations provide details about ions kinetic properties locally and on global scales (eg. Fig. 8), an additional tool for coupling plasma properties that will be detected by the light Ion Analyser (LIA) and large scale structure that will imaged by SXI. In light of the results obtained so far, we propose IAPIC, as a global 3D 
electromagnetic kinetic code to simulate the MP, the cusps, and the magnetosheath, which should enhance the science return of space missions like the CSA - ESA SMILE mission.

\section{Summary and Conclusion}

We have utilized a three-dimensional kinetic particle-in-cell code (IAPIC) to determine the size, shape and location of the Earth's magnetopause with a dipole tilt of $-31^{\circ}$ (North hemisphere summer) in response to solar wind conditions of radial $(\mathrm{B}=\mathrm{Bx})$ and non-radial $(|B z|<|B x|<|B y|)$ IMF. These two IMF orientations cover the range of non-radial IMF with a cone angle between 0 and $50^{\circ}$ as considered by Samsonov et al. (2017) related to a magnetopause crossing event recorded by the THEMIS mission on 16 July 2007. The simulations show sunward MP expansions and predict asymmetric magnetosphere for both IMF orientations.

Using a reference MP size $\left(10.3 R_{E}\right)$ independently derived from (Shue et al., 1997) and based on our pressure balance calculations (Table 4), the findings of this study are summarized as follows:

1. For purely radial IMF, the MP expands from 10.3 to $10.8 R_{E}$ for the full bulk flow. The expansion is limited to only $10.5 R_{E}$ when the effect of the backstreaming ions are dropped from calculations along OX axis. In other words, $40 \%$ of the observed expansion is due to backstreaming solar wind particles. Moreover, along tilted magnetic axis, the MP expansion is from 10.3 to 11.9 and 11.5 with and without backstreaming ion effect, respectively.

2. For non-radial IMF, the expansion is from 10.3 to 11.7 (along $\mathrm{Ox}$ ) and to $12 R_{E}$ (along tilted axis) for the full bulk flow. It is limited to only 11.3 (along Ox) and $11.6 R_{E}$ (along tilted axis) when backstreaming ions are not accounted for in the flow.

3. For non-radial IMF and full bulk flow (i.e., including the effect of backstreaming ions), the expansion rate is 1.4 and $1.7 R_{E}$ along $\mathrm{OX}$ and tilted magnetic equatorial axis, respectively. These rates are comparable with the average expansion rate of 
1.3-1.5 $R_{E}$ reported from THEMIS observations (Jelínek et al., 2010; Suvorova et al., 2010), without being forced to modify the input solar wind parameters as done by MHD model (Samsonov et al., 2017).

4. The backstreaming ions contribution to the bulk flow is $16.5 \%$, and $20 \%$ for radial and non-radial IMF, respectively along the OX axis in the dayside magnetosphere.

5. When deriving the MP position using the maximum density steepening method, the backstreaming ions (characterized and quantified in this study) do not impact the measured size of the MP for both IMF orientations along both OX and tilted axis since they represent only a small percentage of the solar wind plasma.

6. In contrast, when the magnetopause is derived using pressure system balance without accounting the effect of backstreaming ions, the magnetopause expansion is reduced by $0.3-0.4 R_{E}$ (Table 4 ).

7. The difference between magnetopause derivation using maximum density steepening (Garcia \& Hughes, 2007; J. Lu et al., 2015) and the pressure systems balance using definition of dynamic pressure as in (e.g., Willis, 1978, Eq. 3) is consistent with the conclusion drawn by (Samsonov et al., 2020) that density and velocity act differently as a component of dynamic pressure in the pressure system balance. While the energetic backstreaming ions have a small impact on the total density, they have a significant contribution to the total dynamic pressure. In our study, we also considered the effect of thermal pressure in the magnetosheath in the pressure balance.

8. At the subsolar point, the magnetopause expansion is larger for the non-radial case than for the radial case (for both OX and tilted axes). However, in the non-radial case both dawn and dusk magnetopause flanks are squeezed toward the Earth which could be due to the reconnection process in these specific conditions (large IMF $B_{y}$, with a clock angle of $77.6^{\circ}$, a cone angle of $54^{\circ}$ and a large dipole tilt of $31^{\circ}$ corresponding to a North summer time) rarely investigated in past studies. The detailed investigation of the magnetic reconnection process in such conditions is out of the scope of the present work and will be carried out in a further study. 
9. We present new results showing the magnetopause shape in spherical polar coordinates for the two IMF directions. This new technique along with the magnetopause derivations given in Table 4 and Fig. 4 enables us to anticipate the sizes, shapes and locations of magnetopause for all magnetized planets, including magnetized exoplanets. Additionally, this technique accounts for the backstreaming ion contribution to the data used to derive the magnetopause shape, a key information that is not available with other types of simulations.

10. The current study enabled us to derive the solar wind temperature anisotropy, thus paving a research road to study kinetic microinstabilities in the solar wind-magnetosphere coupling (see Figs. 10). In the dayside sector (between -18 and $-10 R_{E}$ along OX) for non-radial IMF, $T_{\perp} / T_{\|}$is large and equal $\approx 6$ for ions and $\approx 1.8$ for electrons. For radial IMF, $T_{\perp} / T_{\|}$equals to 2 . and 2.3 for ions and electrons, respectively. The $T_{\perp e} / T_{\| e}$ ratio can be employed to predicts whistler waves generated by temperature anisotropy like those observed by MMS (e.g., Le Contel et al., 2016). Temperature anisotropy is known to have important effects on the magnetic reconnection process (e.g., Gingell et al., 2015).

11. For both IMF orientations, the magnetopause is found asymmetric: its expansion is larger on the duskside than on the dawnside.

12. In light of the results so far obtained, our findings are considered an additional and key modeling supports to future near-Earth exploration projects, in addition to outer planets' moons and magnetospheres. For example, the newly developed technique to map the MP shape and position, or the ability to track backstreaming ions and their characterizations and their kinetic impact on the plasma flow will contribute to deepen our understanding about the kinetic behavior of plasma in the solar wind magnetosphere coupling and the study of exoplanets magnetospheres.

\section{Future Directions}

Radial and non-radial conditions are relatively infrequent configurations of the IMF at 
Earth, but closer to the Sun, the Parker spiral would favor more and more non-radial IMF orientation (Chang et al., 2019). This suggests that radial IMF conditions are more common at Mercury, which has recently been investigated by MESSENGER and will soon be visited by the BepiColombo spacecraft. Furthermore, Mercury's magnetosphere is much smaller as the magnetopause standoff position is only at about $2 R_{M}$ ( $R_{M}$ being the Mercury radius) and the ion gyroradius is about the size of the planet. Finite Larmor radius effects are expected to play an ever more important role than in the Earth's case (e.g., Johnson et al., 2014; Paral \& Rankin, 2013). Mercury is therefore a natural laboratory for investigating radial IMF and related kinetic effects and we will prepare simulations in advance of BepiColombo's arrival at Mercury. Planets even closer to their stars are common in the galaxy (NASA Exoplanets Archive doi $=10.26133 / N E A 2)$, suggesting that, particularly around cooler M- and Ktype stars, radial IMF may be a common condition. This impacts the structure of their magnetospheres and may influence the escape of planetary atmospheric and ionospheric constituents over time. The kinetic aspect of our approach is particularly sensitive to the dynamics of the bow shock, which may be highly variable in the neighborhood of a small star (Cohen et al., 2015), potentially producing accelerated particles and observable radio emissions (Cohen et al., 2018). One more issue that will be considered for near future work is the impact of the magnetosphere-ionosphere-magnetosheath coupling on magnetopause location. We have tracked in the past $\mathrm{H}^{+}$and $\mathrm{O}^{+}$ions outflow from the ionospheric origin in the dayside magnetosphere (S. M. Baraka \& Ben-Jaffel, 2015). IAPIC can also be used to study the outflow of plasmasphere low energy ions.

\section{Acknowledgments}

This work is carried out using binary data generated by IAPIC, a pic code that is a modified version of the Tristan code that is available to the public through GitHub: https:// github.com/ntoles/tristan-mp-pitp. THEMIS, ACE, and, Wind data are available at http://amda.cdpp.eu/. The used data for generating results is available at ( (https://doi .org/10.48392/bar-001). S Baraka acknowledges the NIA NASA grant \# NNX15AE05G 
806

for supporting this research via the Living and Breathing Planet project. L. Ben-Jaffel acknowledges support from CNES under project PACES. S. Baraka thanks IAP-CNRS for using their computing resources facilities and generating the simulation data. Authors thanks the anonymous reviewers for their critical review and their important remarks that enhanced the quality of this paper. 


\section{References}

Akasofu, S.-I. (1991). Development of magnetospheric physics. Magnetospheric Substorms, $64,3-9$.

Akasofu, S.-I., Roederer, M., \& Krimigis, S. (1982). Dawn-dusk asymmetry of the tail region of the magnetosphere of saturn and the interplanetary magnetic field. Planetary and Space Science, 30(10), 1061-1063.

Artemyev, A., \& Zelenyi, L. (2012, December). Kinetic Structure of Current Sheets in the Earth Magnetotail. Space Science Reviews, 178(2-4), 419-440. doi: 10.1007/ s11214-012-9954-5

Asbridge, J., Bame, S., \& Strong, I. (1968). Outward flow of protons from the earth's bow shock. Journal of Geophysical Research, 73(17), 5777-5782.

Baraka, S. (2016, April). Large Scale Earth's Bow Shock with Northern IMF as Simulated by PIC Code in Parallel with MHD Model. Journal of Astrophysics and Astronomy, 37(2), 1-16. doi: 10.1007/s12036-016-9389-6

Baraka, S., \& Ben-Jaffel, L. (2007, June). Sensitivity of the Earth's magnetosphere to solar wind activity: Three-dimensional macroparticle model. Journal of Geophysical Research (Space Physics), 112, 6212. doi: 10.1029/2006JA011946

Baraka, S., \& Ben-Jaffel, L. (2011). Impact of solar wind depression on the dayside magnetosphere under northward interplanetary magnetic field. Annales Geophysicae, 29(1), 31-46. Retrieved from https://www.ann-geophys.net/29/31/2011/ doi: 10.5194/angeo-29-31-2011

Baraka, S. M., \& Ben-Jaffel, L. (2015). Magnetospheric dynamical and morphological response to multi-species plasma supply from the ionosphere: New comprehensive $3 \mathrm{~d}$ pic simulation. AGUFM, 2015, SM23B-2552.

Ben-Jaffel, L., \& Ballester, G. (2013, May). Hubble Space Telescope detection of oxygen in the atmosphere of exoplanet HD 189733b. Astronomy \& Astrophysics, 553, A52. doi: 10.1051/0004-6361/201221014

Ben-Jaffel, L., \& Ballester, G. E. (2014, April). Transit of Exomoon Plasma Tori: New Diagnosis. \apjl, 785, L30. doi: 10.1088/2041-8205/785/2/L30

Berchem, J., Richard, R. L., Escoubet, C. P., Wing, S., \& Pitout, F. (2016, January). Asymmetrical response of dayside ion precipitation to a large rotation of the imf. Journal of Geophysical Research (Space Physics), 121, 263-273. Retrieved from https:// ui.adsabs.harvard.edu/abs/2016JGRA . 121 . 263B doi: 10.1002/2015JA021969

Blanco-Cano, X., Omidi, N., \& Russell, C. (2006). Macrostructure of collisionless bow shocks: 2. ULF waves in the foreshock and magnetosheath. Journal of Geophysical Research: Space Physics (1978-2012), $111(\mathrm{~A} 10)$.

Blanco-Cano, X., Omidi, N., \& Russell, C. (2009). Global hybrid simulations: Foreshock waves and cavitons under radial interplanetary magnetic field geometry. Journal of Geophysical Research: Space Physics, $114(\mathrm{~A} 1)$.

Bobra, M., Petrinec, S., Fuselier, S., Claflin, E., \& Spence, H. E. (2004). On the solar wind control of cusp aurora during northward imf. Geophysical research letters, $31(4)$.

Bonifazi, C., \& Moreno, G. (1981a). Reflected and diffuse ions backstreaming from the earth's bow shock 1. basic properties. Journal of Geophysical Research: Space Physics, 86, 4397-4404. doi: 10.1029/ja086ia06p04397

Bonifazi, C., \& Moreno, G. (1981b). Reflected and diffuse ions backstreaming from the Earth's bow shock 2. Origin. Journal of Geophysical Research: Space Physics (19782012), 86(A6), 4405-4413.

Bonifazi, C., Moreno, G., Lazarus, A. J., \& Sullivan, J. D. (1980, November). Deceleration of the solar wind in the earth's foreshock region - ISEE 2 and IMP 8 observations. textbackslashjgr, 85, 6031-6038. doi: 10.1029/JA085iA11p06031

Brackbill, J. U. (2011). A comparison of fluid and kinetic models of steady magnetic reconnection. Physics of Plasmas (1994-present), 18(3), 032309. doi: 10.1063/1 .3568828

Buneman, O., Neubert, T., \& Nishikawa, K.-I. (1992). Solar wind-magnetosphere interaction as simulated by a 3 -d em particle code. IEEE transactions on plasma science, 20(6), 
810-816.

Cai, D., Esmaeili, A., Lembège, B., \& Nishikawa, K.-I. (2015). Cusp dynamics under northward imf using three-dimensional global particle-in-cell simulations. Journal of Geophysical Research: Space Physics, 120(10), 8368-8386.

Cai, D., Li, Y., Nishikawa, K.-I., Xiao, C., Yan, X., \& Pu, Z. (2003). Parallel 3-D Electromagnetic Particle Code Using High Performance FORTRAN: Parallel TRISTAN. In Space Plasma Simulation (Vol. 615, pp. 25-53). Springer Berlin Heidelberg. doi: 10.1007/3-540-36530-3_2

Chang, Q., Xu, X., Xu, Q., Zhong, J., Xu, J., Wang, J., \& Zhang, T. (2019). Multiple-point modeling the parker spiral configuration of the solar wind magnetic field at the solar maximum of solar cycle 24. The Astrophysical Journal, 884(2), 102.

Cohen, O., Ma, Y., Drake, J. J., Glocer, A., Garraffo, C., Bell, J. M., \& Gombosi, T. I. (2015, jun). THE INTERACTION OF VENUS-LIKE, m-DWARF PLANETS WITH THE STELLAR WIND OF THEIR HOST STAR. The Astrophysical Journal, 806(1), 41. Retrieved from https://doi.org/10.1088/0004-637x/806/1/41 doi: 10.1088/ 0004-637x/806/1/41

Cohen, O., Moschou, S.-P., Glocer, A., Sokolov, I. V., Mazeh, T., Drake, J. J., ... AlvaradoGómez, J. D. (2018, oct). Exoplanet modulation of stellar coronal radio emission. The Astronomical Journal, 156(5), 202. Retrieved from https://doi.org/10.3847/ 1538-3881/aae1f2 doi: 10.3847/1538-3881/aae1f2

Dimmock, A., Nykyri, K., Osmane, A., Karimabadi, H., \& Pulkkinen, T. (2017). Dawndusk asymmetries of the earth's dayside magnetosheath in the magnetosheath interplanetary medium reference frame. Dawn-Dusk Asymmetries in Planetary Plasma Environments, 49-72.

Dimmock, A. P., Nykyri, K., Karimabadi, H., Osmane, A., \& Pulkkinen, T. I. (2015, April). A statistical study into the spatial distribution and dawn-dusk asymmetry of dayside magnetosheath ion temperatures as a function of upstream solar wind conditions. Journal of Geophysical Research (Space Physics), 120(4), 2767-2782. doi: 10.1002/ 2014JA020734

Dimmock, A. P., Nykyri, K., \& Pulkkinen, T. I. (2014, August). A statistical study of magnetic field fluctuations in the dayside magnetosheath and their dependence on upstream solar wind conditions. Journal of Geophysical Research (Space Physics), 119(8), 6231-6248. doi: 10.1002/2014JA020009

Dušík, Š., Granko, G., Šafránková, J., Němeček, Z., \& Jelínek, K. (2010). Imf cone angle control of the magnetopause location: Statistical study. Geophysical Research Letters, $37(19)$.

Eastwood, J. P. (2008). The science of space weather. Philosophical Transactions of the Royal Society A: Mathematical, Physical and Engineering Sciences, 366(1884), 44894500 .

Fairfield, D., Baumjohann, W., Paschmann, G., Lühr, H., \& Sibeck, D. (1990). Upstream pressure variations associated with the bow shock and their effects on the magnetosphere. Journal of Geophysical Research: Space Physics, 95(A4), 3773-3786.

Garcia, K., \& Hughes, W. (2007). Finding the lyon-fedder-mobarry magnetopause: A statistical perspective. Journal of Geophysical Research: Space Physics, 112(A6).

Gingell, P. W., Burgess, D., \& Matteini, L. (2015, March). The Three-dimensional Evolution of Ion-scale Current Sheets: Tearing and Drift-kink Instabilities in the Presence of Proton Temperature Anisotropy. The Astrophysical Journal, 802(1), 4. doi: 10.1088/ 0004-637X/802/1/4

Greenstadt, E. W., Green, I. M., Inouye, G. T., Hundhausen, A. J., Bame, S. J., \& Strong, I. B. (1968, January). Correlated magnetic field and plasma observations of the Earth's bow shock. \textbackslashjgr, 73, 51. doi: 10.1029/JA073i001p00051

Grygorov, K., Šafránková, J., Němeček, Z., Pi, G., Přech, L., \& Urbář, J. (2017). Shape of the equatorial magnetopause affected by the radial interplanetary magnetic field. Planetary and Space Science, 148, 28-34.

Haaland, S., Lybekk, B., Maes, L., Laundal, K., Pedersen, A., Tenfjord, P., ... Snekvik, 
K. (2017). North-south asymmetries in cold plasma density in the magnetotail lobes: Cluster observations. Journal of Geophysical Research: Space Physics, 122(1), 136149.

Haaland, S., Reistad, J., Tenfjord, P., Gjerloev, J., Maes, L., DeKeyser, J., ... Dorville, N. (2014). Characteristics of the flank magnetopause: Cluster observations. Journal of Geophysical Research: Space Physics, 119(11), 9019-9037.

Haaland, S., Runov, A., Artemyev, A., \& Angelopoulos, V. (2019). Characteristics of the flank magnetopause: Themis observations. Journal of Geophysical Research: Space Physics, 124(5), 3421-3435.

Heikkila, W. J. (2011). Earth's Magnetosphere: Formed by the Low-latitude Boundary Layer. Elsevier.

Jacobsen, K. S., Phan, T. D., Eastwood, J. P., Sibeck, D. G., Moen, J. I., Angelopoulos, V., ... Fornaçon, K.-H. (2009). THEMIS observations of extreme magnetopause motion caused by a hot flow anomaly. Journal of Geophysical Research (Space Physics), 114, 8210. Retrieved from http://adsabs.harvard.edu/abs/2009JGRA. . 114.8210J

Jelínek, K., Němeček, Z., Šafránková, J., Shue, J.-H., Suvorova, A. V., \& Sibeck, D. G. (2010). Thin magnetosheath as a consequence of the magnetopause deformation: THEMIS observations. Journal of Geophysical Research (Space Physics), 115, 10203. Retrieved from http://adsabs.harvard.edu/abs/2010JGRA. . 11510203J

Johnson, J. R., Wing, S., \& Delamere, P. A. (2014). Kelvin helmholtz instability in planetary magnetospheres. Space Science Reviews, 184(1-4), 1-31.

Karimabadi, H., Krauss-Varban, D., Huba, J., \& Vu, H. (2004). On magnetic reconnection regimes and associated three-dimensional asymmetries: Hybrid, hall-less hybrid, and hall-mhd simulations. Journal of Geophysical Research: Space Physics, 109(A9).

Karimabadi, H., Roytershteyn, V., Vu, H. X., Omelchenko, Y. A., Scudder, J., Daughton, W., ... Geveci, B. (2014). The link between shocks, turbulence, and magnetic reconnection in collisionless plasmas. Physics of Plasmas, 21, 062308. doi: 10.1063/ 1.4882875

Lavraud, B., Borovsky, J. E., Génot, V., Schwartz, S. J., Birn, J., Fazakerley, A. N., ... Wild, J. A. (2009). Tracing solar wind plasma entry into the magnetosphere using ion-to-electron temperature ratio. Geophys. Res. Lett., 36, 18109. Retrieved from http://adsabs . harvard. edu/abs/2009GeoRL. . 3618109L

Le Contel, O., Retinò, A., Breuillard, H., Mirioni, L., Robert, P., Chasapis, A., ... Saito, Y. (2016, June). Whistler mode waves and Hall fields detected by MMS during a dayside magnetopause crossing. Geophysical Research Letters, 43(12), 5943-5952. doi: $10.1002 / 2016$ GL068968

Lindman, E. (1975). "Free-space" boundary conditions for the time dependent wave equation. J. Comput. Phys., 18(1), 66-78.

Liu, Y.-H., Li, T., Hesse, M., Sun, W., Liu, J., Burch, J., ... Huang, K. (2019). Threedimensional magnetic reconnection with a spatially confined x-line extent: Implications for dipolarizing flux bundles and the dawn-dusk asymmetry. Journal of Geophysical Research: Space Physics, 124(4), 2819-2830.

Lu, J., Wang, M., Kabin, K., Zhao, J., Liu, Z.-Q., Zhao, M., \& Li, G. (2015). Pressure balance across the magnetopause: Global mhd results. Planetary and Space Science, $106,108-115$.

Lu, S., Lin, Y., Angelopoulos, V., Artemyev, A., Pritchett, P., Lu, Q., \& Wang, X. (2016). Hall effect control of magnetotail dawn-dusk asymmetry: A three-dimensional global hybrid simulation. Journal of Geophysical Research: Space Physics, 121(12), 11-882.

Luo, H., Chen, G., Du, A., \& Xu, W. (2013). Solar wind dependence of energy coupling between solar wind and magnetosphere during intense northward imfs. Planetary and Space Science, 79, 82-89.

Masters, A., Slavin, J., DiBraccio, G., Sundberg, T., Winslow, R., Johnson, C., ... Korth, H. (2013). A comparison of magnetic overshoots at the bow shocks of mercury and saturn. Journal of Geophysical Research: Space Physics, 118(7), 4381-4390.

Merka, J., Szabo, A., Šafránková, J., \& Němeček, Z. (2003). Earth's bow shock and 
magnetopause in the case of a field-aligned upstream flow: Observation and model comparison. Journal of Geophysical Research: Space Physics (1978-2012), 108(A7), -.

Němeček, Z., Šafránková, J., Zastenker, G. N., Pišoft, P., \& Jelínek, K. (2002, April). Low-frequency variations of the ion flux in the magnetosheath. Planetary and Space Science, 50(5-6), 567-575. doi: 10.1016/S0032-0633(02)00036-3

Omidi, N., Blanco-Cano, X., Russell, C. T., \& Karimabadi, H. (2004, January). Dipolar magnetospheres and their characterization as a function of magnetic moment. Advances in Space Research, 33, 1996-2003. doi: 10.1016/j.asr.2003.08.041

Østgaard, N., Mende, S., Frey, H., Frank, L., \& Sigwarth, J. (2003). Observations of non-conjugate theta aurora. Geophysical Research Letters, $30(21)$.

Palmroth, M., Ganse, U., Pfau-Kempf, Y., Battarbee, M., Turc, L., Brito, T., ... von Alfthan, S. (2018, August). Vlasov methods in space physics and astrophysics. Living Reviews in Computational Astrophysics, 4(1), 1. doi: 10.1007/s41115-018-0003-2

Paral, J., \& Rankin, R. (2013). Dawn-dusk asymmetry in the kelvin-helmholtz instability at mercury. Nature Communications, 4(1), 1-5.

Park, J.-S., Shue, J.-H., Kim, K.-H., Pi, G., Němeček, Z., \& Å afránková, J. (2016, July). Global expansion of the dayside magnetopause for long-duration radial IMF events: Statistical study on GOES observations. Journal of Geophysical Research (Space Physics), 121(7), 6480-6492. doi: 10.1002/2016JA022772

Parks, G. K. (1991). Physics of space plasmas-an introduction. Redwood City, CA, AddisonWesley Publishing Co., 1991, 547 p..

Paschmann, G., Sckopke, N., Papamastorakis, I., Asbridge, J., Bame, S., \& Gosling, J. (1981). Characteristics of reflected and diffuse ions upstream from the earth's bow shock. Journal of Geophysical Research: Space Physics (1978-2012), 86(A6), 43554364 .

Paularena, K., Richardson, J., Kolpak, M., Jackson, C., \& Siscoe, G. (2001). A dawn-dusk density asymmetry in earth's magnetosheath. Journal of Geophysical Research: Space Physics, 106(A11), 25377-25394.

Pi, G., Němeček, Z., Šafránková, J., Grygorov, K., \& Shue, J.-H. (2018). Formation of the dayside magnetopause and its boundary layers under the radial imf. Journal of Geophysical Research: Space Physics, 123(5), 3533-3547.

Samsonov, A. (2006, January). Numerical modelling of the Earth's magnetosheath for different IMF orientations. Advances in Space Research, 38(8), 1652-1656. doi: 10 $.1016 /$ j.asr.2005.06.009

Samsonov, A. A. (2011, January). Propagation of inclined interplanetary shock through the magnetosheath. Journal of Atmospheric and Solar-Terrestrial Physics, 73, 30-39. doi: $10.1016 /$ j.jastp.2009.10.014

Samsonov, A. A., Bogdanova, Y. V., Branduardi-Raymont, G., Sibeck, D. G., \& Toth, G. (2020). Is the Relation Between the Solar Wind Dynamic Pressure and the Magnetopause Standoff Distance so Straightforward? Geophys. Res. Lett., 47 (8). doi: 10.1029/2019GL086474

Samsonov, A. A., Němeček, Z., Šafránková, J., \& Jelínek, K. (2012, May). Why does the subsolar magnetopause move sunward for radial interplanetary magnetic field? Journal of Geophysical Research (Space Physics), 117, 5221. doi: 10.1029/2011JA017429

Samsonov, A. A., Sibeck, D. G., Å afránková, J., Němeček, Z., \& Shue, J. H. (2017, March). A method to predict magnetopause expansion in radial IMF events by MHD simulations. Journal of Geophysical Research (Space Physics), 122(3), 3110-3126. doi: 10.1002/2016JA023301

Shue, J. H., Chao, J. K., Fu, H. C., Russell, C. T., Song, P., Khurana, K. K., \& Singer, H. J. (1997, May). A new functional form to study the solar wind control of the magnetopause size and shape. Journal of Geophysical Research (Space Physics), 102(A5), 9497-9512. doi: 10.1029/97JA00196

Shue, J.-H., Chao, J.-K., Song, P., McFadden, J., Suvorova, A., Angelopoulos, V., ... Plaschke, F. (2009). Anomalous magnetosheath flows and distorted subsolar mag- 
netopause for radial interplanetary magnetic fields. Geophysical Research Letters, $36(18)$.

Sibeck, D. G., Allen, R., Aryan, H., Bodewits, D., Brandt, P., Branduardi-Raymont, G., ... others (2018). Imaging plasma density structures in the soft x-rays generated by solar wind charge exchange with neutrals. Space Science Reviews, 214(4), 79.

Sibeck, D. G., Decker, R. B., Mitchell, D. G., Lazarus, A. J., Lepping, R. P., \& Szabo, A. (2001). Solar wind preconditioning in the flank foreshock: IMP 8 observations. J. Geophys. Res., 106, 21675-21688. Retrieved from http://adsabs.harvard.edu/ abs/2001JGR . . 10621675S

Sorathia, K., Merkin, V., Ukhorskiy, A., Allen, R., Nykyri, K., \& Wing, S. (2019). Solar wind ion entry into the magnetosphere during northward imf. Journal of Geophysical Research: Space Physics, 124(7), 5461-5481.

Spreiter, J. R., \& Alksne, A. Y. (1969). Plasma flow around the magnetosphere. Reviews of Geophysics, 7(1-2), 11-50.

Suvorova, A., \& Dmitriev, A. (2015). Magnetopause inflation under radial imf: Comparison of models. Earth and Space Science, 2(4), 107-114.

Suvorova, A. V., Shue, J. H., Dmitriev, A. V., Sibeck, D. G., McFadden, J. P., Hasegawa, H., . . Němeček, Z. (2010, October). Magnetopause expansions for quasi-radial interplanetary magnetic field: THEMIS and Geotail observations. Journal of Geophysical Research (Space Physics), 115(A10), A10216. doi: 10.1029/2010JA015404

Tan, B., Lin, Y., Perez, J., \& Wang, X. (2011). Global-scale hybrid simulation of dayside magnetic reconnection under southward imf: Structure and evolution of reconnection. Journal of Geophysical Research: Space Physics, 116(A2).

Trattner, K., Mulcock, J., Petrinec, S., \& Fuselier, S. (2007). Location of the reconnection line at the magnetopause during southward imf conditions. Geophysical Research Letters, $34(3)$.

Treumann, R. A., \& Baumjohann, W. (2013). Collisionless magnetic reconnection in space plasmas. Frontiers in Physics, 1, 31.

Tsurutani, B. T., \& Lakhina, G. S. (1997). Some basic concepts of wave-particle interactions in collisionless plasmas. Reviews of Geophysics, 35 (4), 491-501.

Turc, L., Tarvus, V., Dimmock, A., Battarbee, M., Ganse, U., Johlander, A., ... Palmroth, M. (2020). Asymmetries in the earth's dayside magnetosheath: results from global hybrid-vlasov simulations. Annales Geophysicae Discussions, 2020, 124. Retrieved from https://www. ann-geophys-discuss.net/angeo-2020-13/ doi: 10.5194/angeo-2020-13

Turner, D., Wilson, L., Liu, T., Cohen, I., Schwartz, S., Osmane, A., .. others (2018). Autogenous and efficient acceleration of energetic ions upstream of earth's bow shock. Nature, 561(7722), 206-210.

Villasenor, J., \& Buneman, O. (1992). Rigorous charge conservation for local electromagnetic field solvers. Comput. Phys. Commun., 69(2), 306-316.

Šafránková, J., Němeček, Z., Santolík, O., Sibeck, D. G., Zastenker, G. N., \& Skalsky, A. (2000). The Flank Magnetopause: Interball Observations. Advances in Space Research, 25, 1503-1510. Retrieved from http://adsabs .harvard.edu/abs/2000AdSpR ..25.1503S

Walsh, A. P., Haaland, S., Forsyth, C., Keesee, A. M., Kissinger, J., Li, K., ... Taylor, M. G. G. T. (2014, July). Dawn-dusk asymmetries in the coupled solar windmagnetosphere-ionosphere system: a review. Annales Geophysicae, 32, 705-737. Retrieved from https://ui.adsabs.harvard.edu/abs/2014AnGeo..32..705W doi: 10.5194/angeo-32-705-2014

Walsh, B. M. (2017). Magnetopause plasma parameters and asymmetries in solar windmagnetosphere coupling. Dawn-Dusk Asymmetries in Planetary Plasma Environments, 29-39.

Walsh, B. M., Sibeck, D. G., Wang, Y., \& Fairfield, D. H. (2012, December). Dawn-dusk asymmetries in the Earth's magnetosheath. Journal of Geophysical Research (Space Physics), 117(A12), A12211. doi: 10.1029/2012JA018240 
Wang, C.-P., Lyons, L. R., Weygand, J. M., Nagai, T., \& McEntire, R. W. (2006). Equatorial distributions of the plasma sheet ions, their electric and magnetic drifts, and magnetic fields under different interplanetary magnetic field bz conditions. Journal of Geophysical Research: Space Physics, 111(A4).

Wang, J., Guo, Z., Ge, Y. S., Du, A., Huang, C., \& Qin, P. (2018, October). The responses of the earth's magnetopause and bow shock to the IMF $\mathrm{B}_{z}$ and the solar wind dynamic pressure: a parametric study using the AMR-CESE-MHD model. Journal of Space Weather and Space Climate, 8, A41. doi: 10.1051/swsc/2018030

Wang, Y.-M., Grappin, R., Robbrecht, E., \& Sheeley Jr, N. (2012). The Astrophysical Journal, $749(2), 182$.

Willis, D. (1978). The magnetopause: Microstructure and interaction with magnetospheric plasma. Journal of Atmospheric and Terrestrial Physics, 40(3), 301-322.

Wing, S., Johnson, J. R., Chaston, C. C., Echim, M., Escoubet, C. P., Lavraud, B., ... Wang, C. P. (2014, November). Review of Solar Wind Entry into and Transport Within the Plasma Sheet. Space Science Reviews, 184(1-4), 33-86. doi: 10.1007/ s11214-014-0108-9

Yu, Y., \& Ridley, A. J. (2009, December). The response of the magnetosphere-ionosphere system to a sudden dynamic pressure enhancement under southward IMF conditions. Annales Geophysicae, 27(12), 4391-4407. doi: 10.5194/angeo-27-4391-2009

Zhang, H., Fu, S., Pu, Z., Lu, J., Zhong, J., Zhu, C., ... Liu, L. (2019, aug). Statistics on the magnetosheath properties related to magnetopause magnetic reconnection. The Astrophysical Journal, 880(2), 122. Retrieved from https://doi.org/ 10.3847\%2F1538-4357\%2Fab290e doi: 10.3847/1538-4357/ab290e 


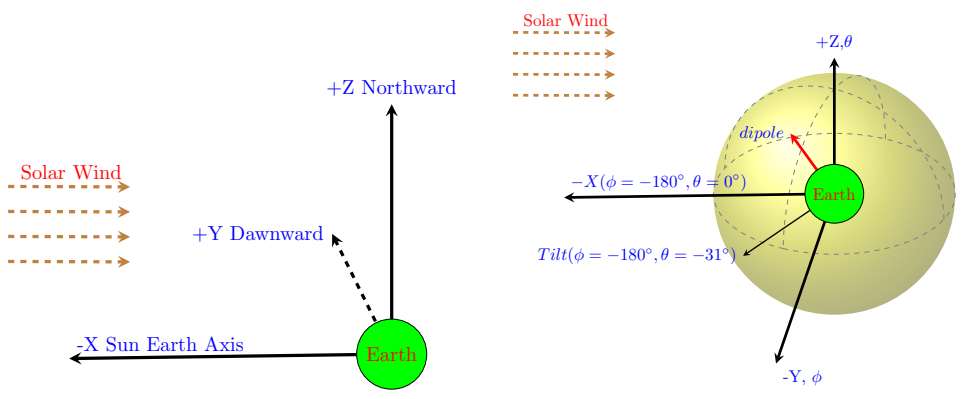

1

Figure 1. Code coordinates scheme (left) and spherical coordinates illustration used in MP derivation (right) 

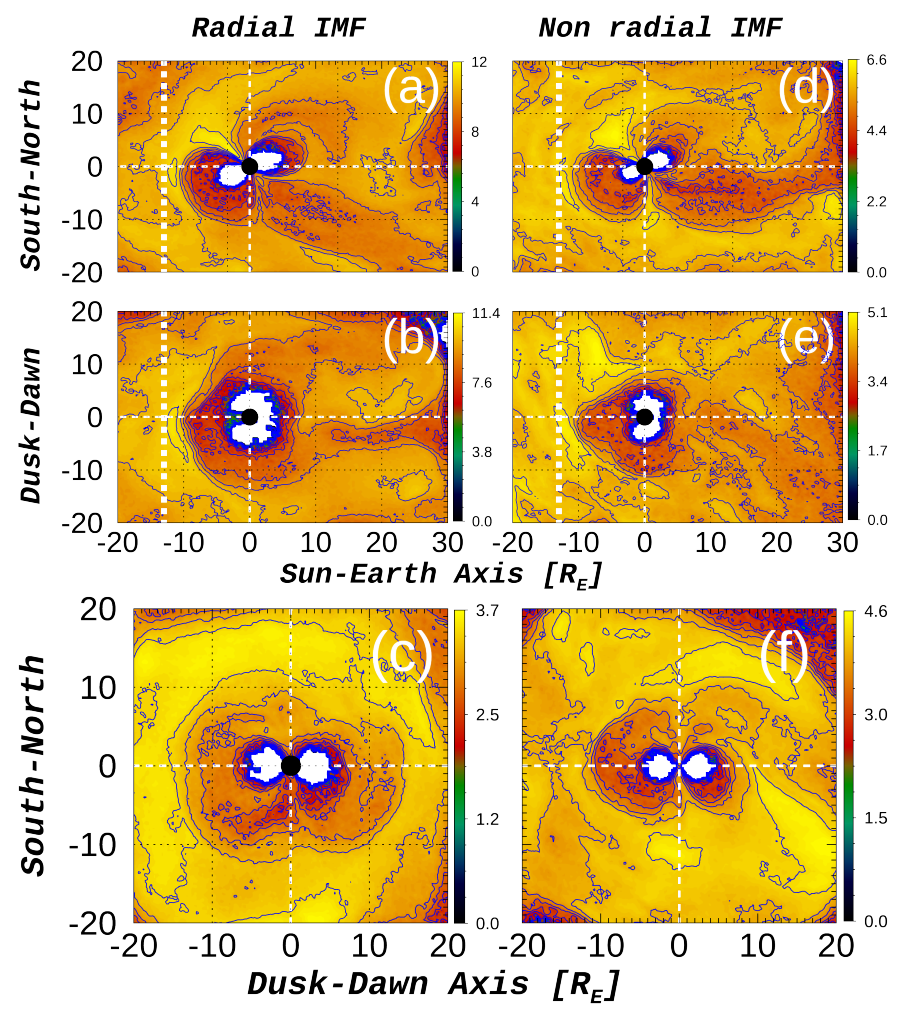

Figure 2. Solar wind $2 \mathrm{D}$ density contours in the $\mathrm{XZ}(\mathrm{Y}=0), \mathrm{XY}(\mathrm{Z}=0)$, and $\mathrm{YZ}(\mathrm{X}=0)$ planes for both IMF orientations. Vertical dashed lines at $X=-13 R_{E}$ indicate the position of linear density profiles shown in Fig. 8. Panels (a), (b), and (c) are for radial IMF and panels (d), (e), and (f) are for non-radial IMF

This article is protected by copyright. All rights reserved. 

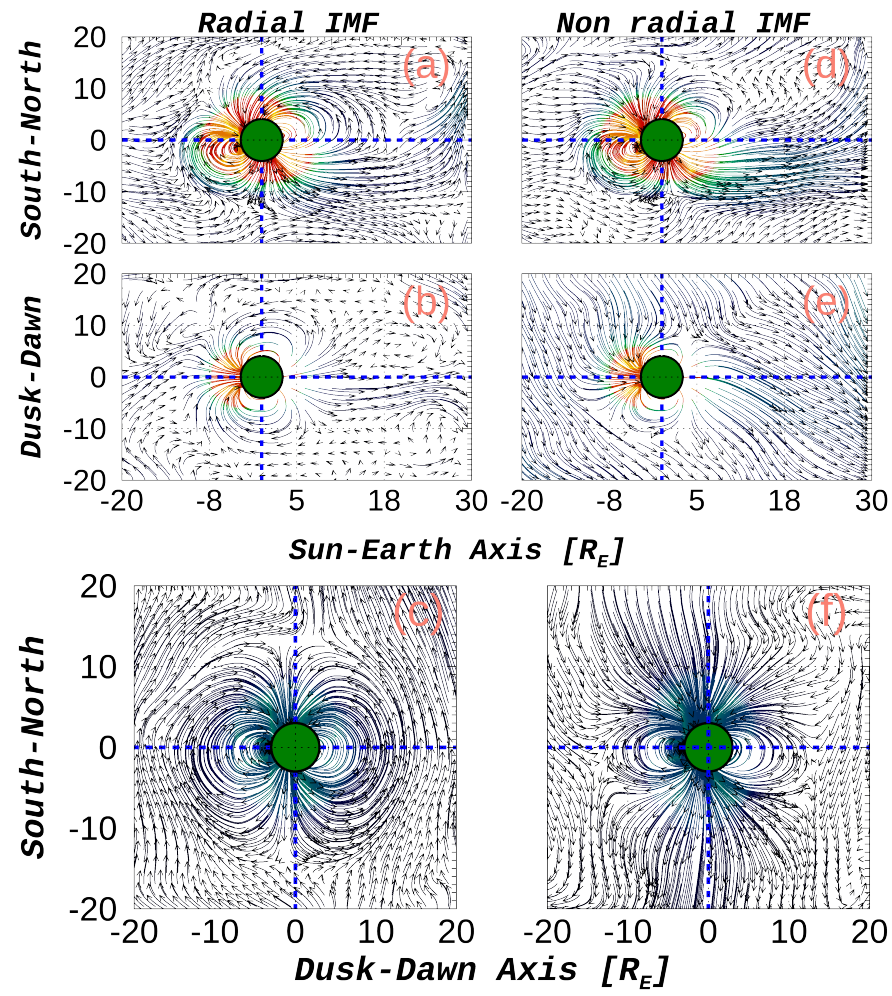

Figure 3. Fields 2D topology in the $\mathrm{XZ}(\mathrm{Y}=0), \mathrm{XY}(\mathrm{Z}=0)$, and $\mathrm{YZ}(\mathrm{X}=0)$ planes for both IMF orientations. Panels (a), (b), and (c) are for radial IMF and panels (d), (e), and (f) are for non radial IMF 

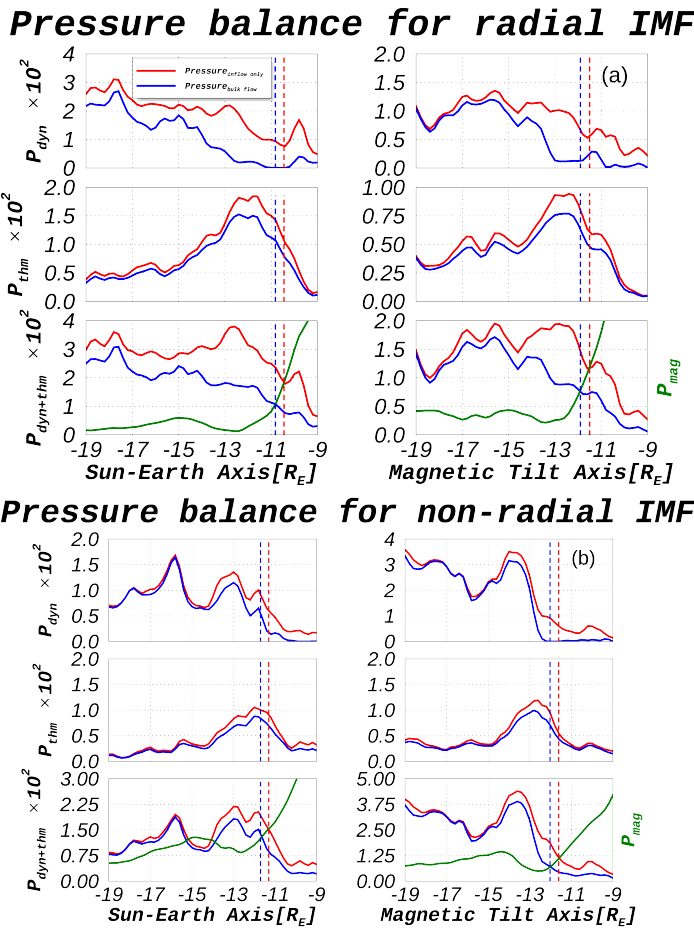

Figure 4. MP size derivation from pressure balance system measured radially at two locations. The MP is derived along the Earth-Sun axis (OX) and along the tilted magnetic equatorial axis. Bulk flow (blue) and inflow (red) pressures show different MP locations. For radial IMF, panel (a), it is found that $\mathrm{MP}=[-10.8,-10.5] R_{E}$ derived with bulk and inflow pressures only along Sun-Earth Axis and $\mathrm{MP}=[-11.9,-11.5] R_{E}$ along the tilted magnetic equatorial axis. Respectively for non-radial $\mathrm{IMF}, \operatorname{panel}(\mathrm{b}), \mathrm{MP}=[-11.7,-11.3]$ and $[-12 .,-11.6] R_{E}$. 

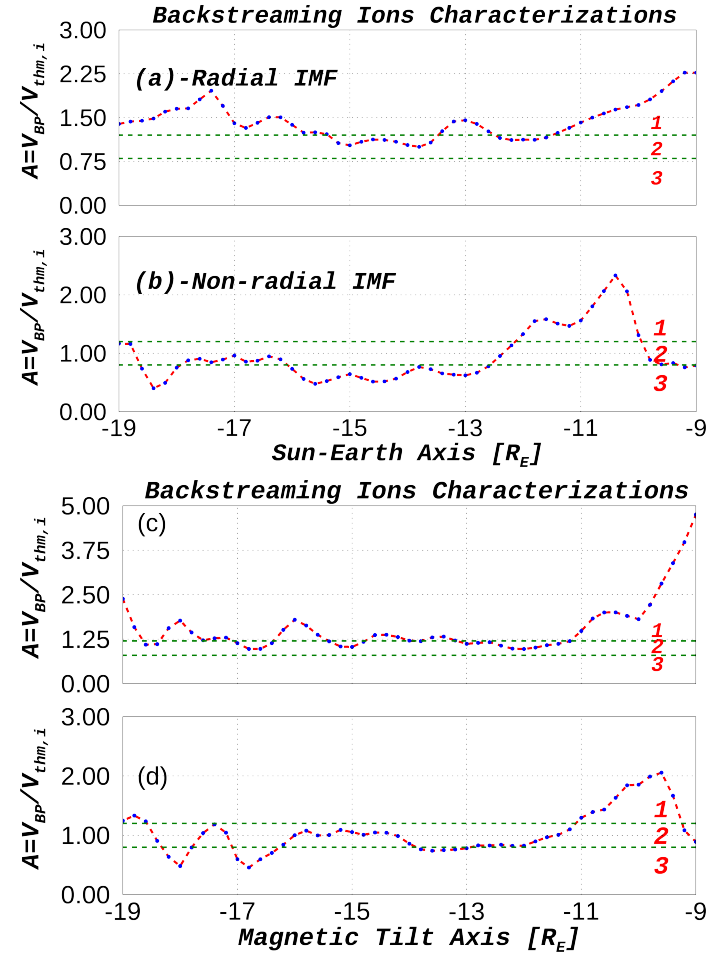

Figure 5. Characterization of backstreaming ions in the dayside magnetosphere based on their bulk flow ratio on thermal speed (Adopted from (Bonifazi \& Moreno, 1981b)). Panels, a and $\mathrm{b}$ are taken along $\mathrm{OX}$-axis in XZ plane and panels $\mathrm{c}$, and $\mathrm{d}$ are taken along tilted magnetic equatorial axis for both IMF orientations. This figure shows the reflected and diffuse fractions of the backstreaming ions (their respective percentages are shown in table 5). Scaling factor "A" is used for the characterization of backstreaming ions. $\mathrm{A}>1.2$ denotes reflected ions(region- 1 ) in the plots. $\mathrm{A}<0.8$ denotes diffuse ions(region-3). $0.8<\mathrm{A}<1.2$ denotes intermediate ions(region-2) 


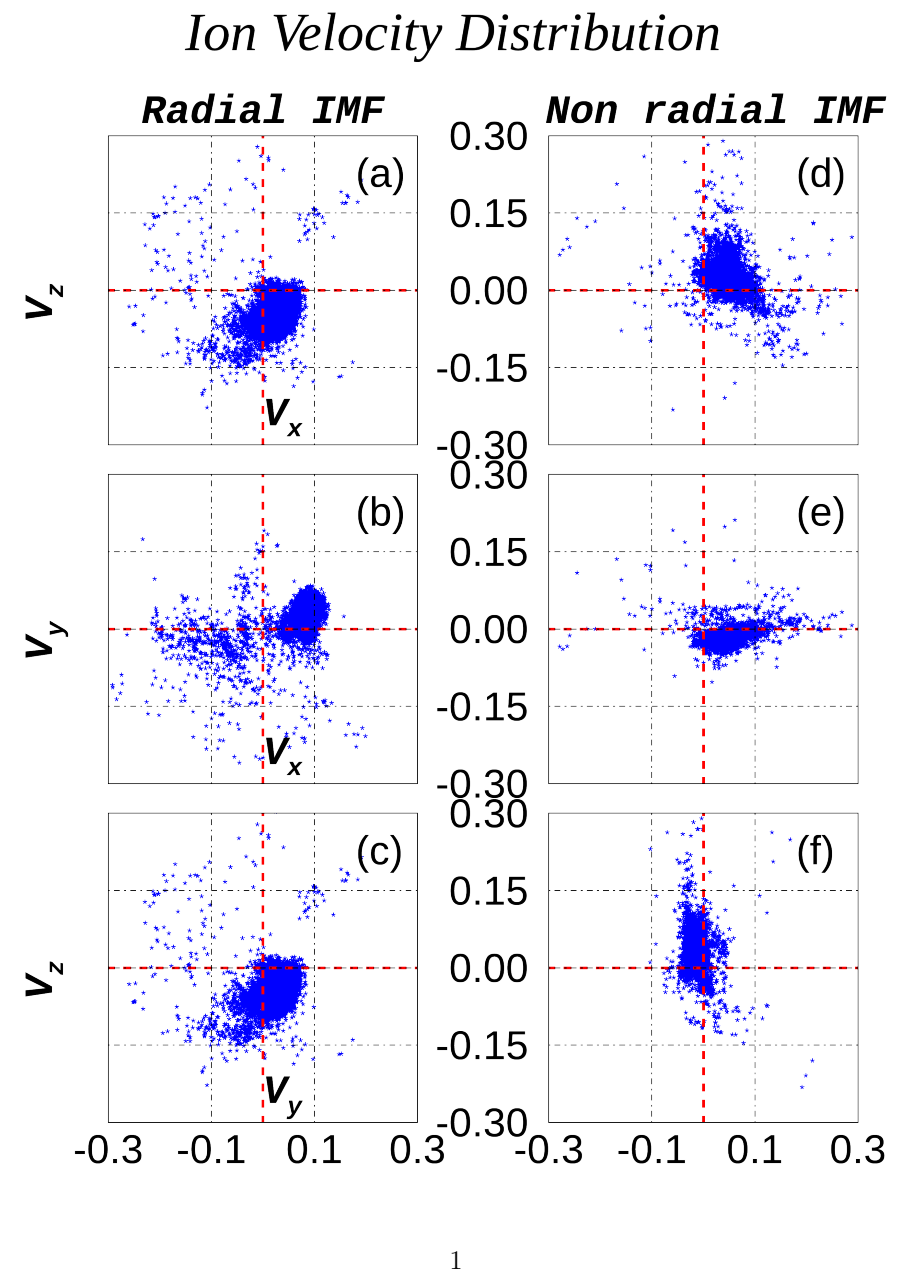

Figure 6. Ion Velocity dayside distribution measured from $-20 R_{E}$ to the planet position $(\mathrm{z}=\mathrm{y}=0)$ along Sun-Earth axis. Left panels are for radial IMF and right panels are for non radial IMF orientation. 


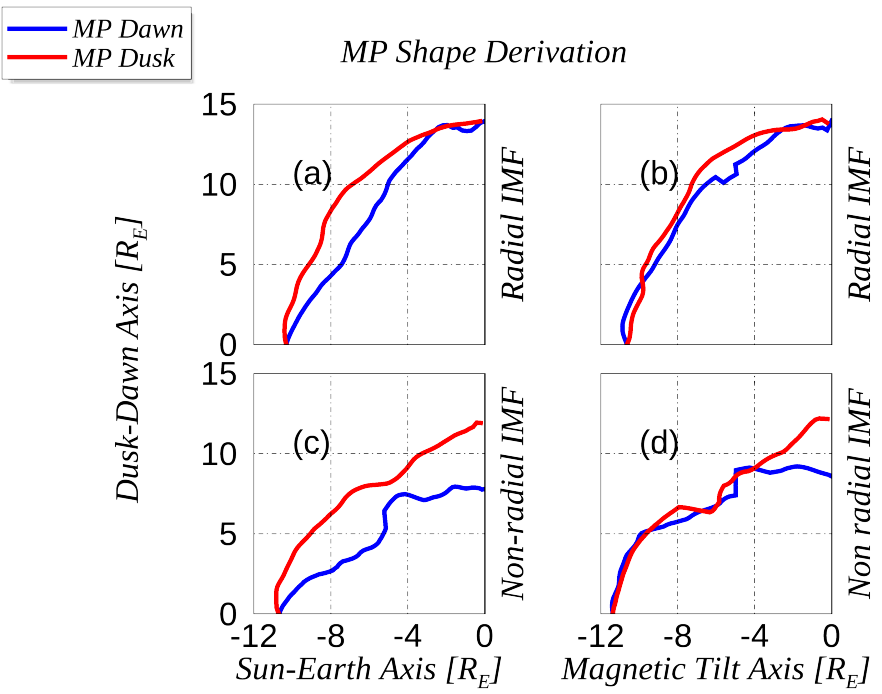

Figure 7. Panels (a) and (b) show the MP shape for radial IMF along Sun-Earth and tilted magnetic equatorial axis. Similarly, panels (c) and (d) are the same but for non-radial IMF. We remark that the size shown here is approximately equivalent to the MP sizes derived from the density steepening. 


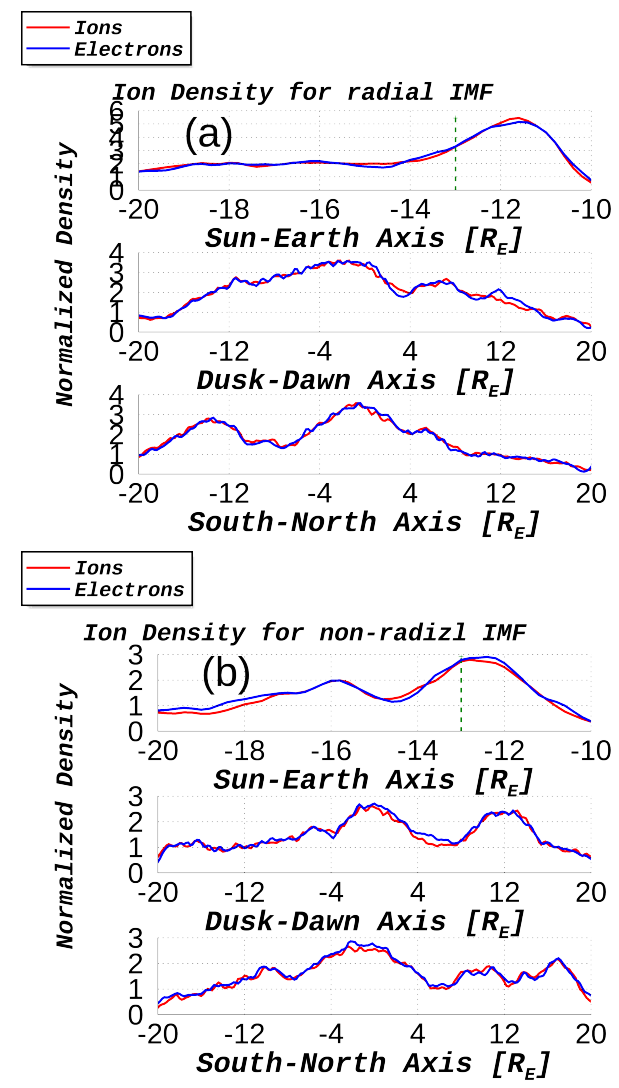

1

Figure 8. Ion and electron densities are plotted in three directions, along $\mathrm{OX}(\mathrm{Y}=\mathrm{Z}=0)$, $\mathrm{OY}$ $(\mathrm{X}=-13$, and $\mathrm{Z}=0)$, and $\mathrm{OZ}(\mathrm{X}=-13$, and $\mathrm{Y}=0)$ ), just outside the MP for radial IMF in panel a and for non-radial IMF in panel $b$. Their values are normalized to the initial densities. The density profile is plotted only in the dayside region as shown with vertical dashed lines $\left(\right.$ at $\left.X=-13 R_{E}\right)$ in Fig. 2. This figure also shows the plasma quasi neutrality.

This article is protected by copyright. All rights reserved. 

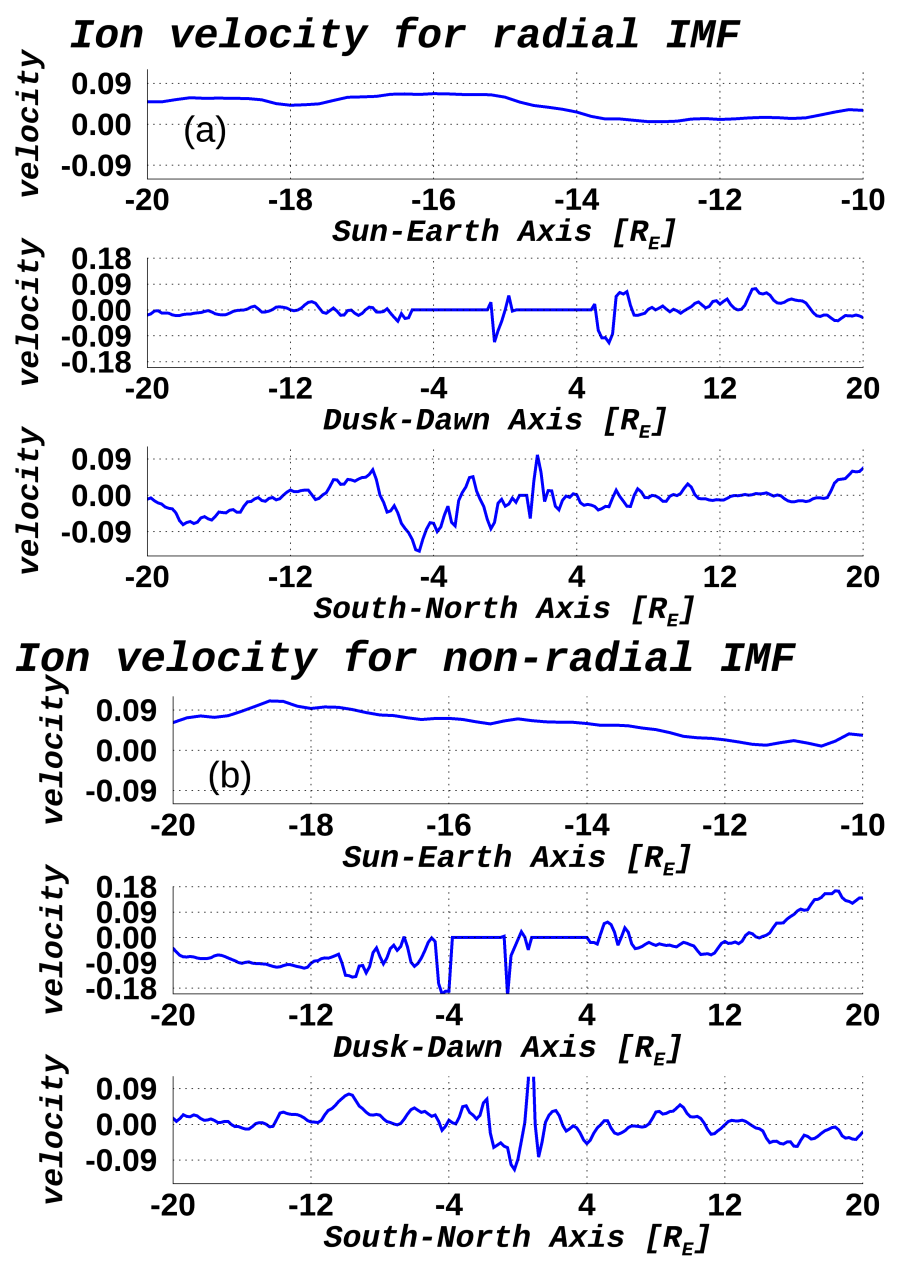

Figure 9. Bulk ion velocity modulus for both IMF orientations are plotted along three directions as in Fig. 8.

This article is protected by copyright. All rights reserved. 

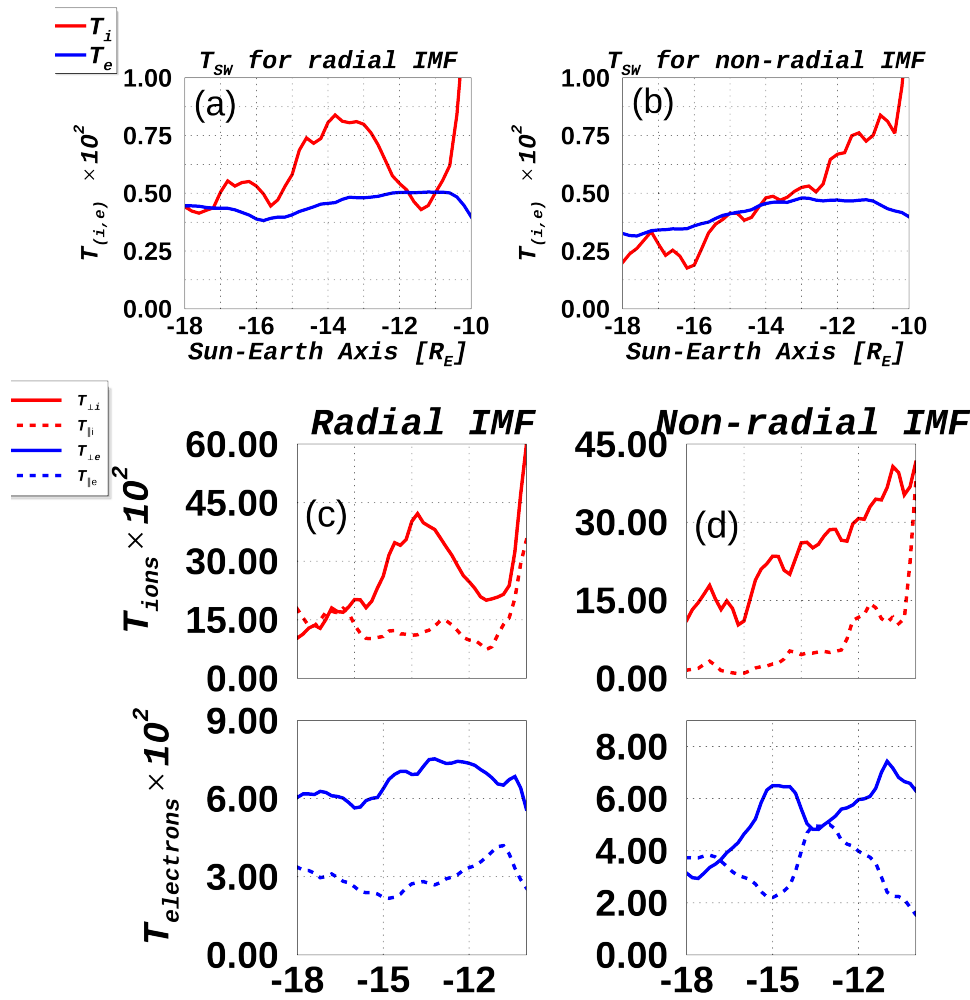

\section{Sun-Earth Axis $\left[R_{E}\right]$}

Figure 10. Ion and electron temperatures are shown in the dayside magnetosphere for both IMF orientations(panels a and $\mathrm{b}$ ). The average ion to electron temperature ratios in the magnetosheath between -14 and $-12.5 R_{E}$ are $\left\langle T_{i} / T_{e}\right\rangle=1.72$ and $\left\langle T_{i} / T_{e}\right\rangle=1.1$ for radial (panel a) and nonradial IMF (panel b), respectively. Temperature anisotropy for ions and electrons for both IMF orientations are shown in panel $\mathrm{c}$ and $\mathrm{d}$. The $T_{\perp i} / T_{\| i}$, and $T_{\perp e} / T_{\| e}$ ratios averaged between -18 to $-10 R_{E}$ are $(2,2.3)$ respectively for radial IMF. This ratio reads $(6,1.8)$ for non-radial IMF.

This article is protected by copyright. All rights reserved. 


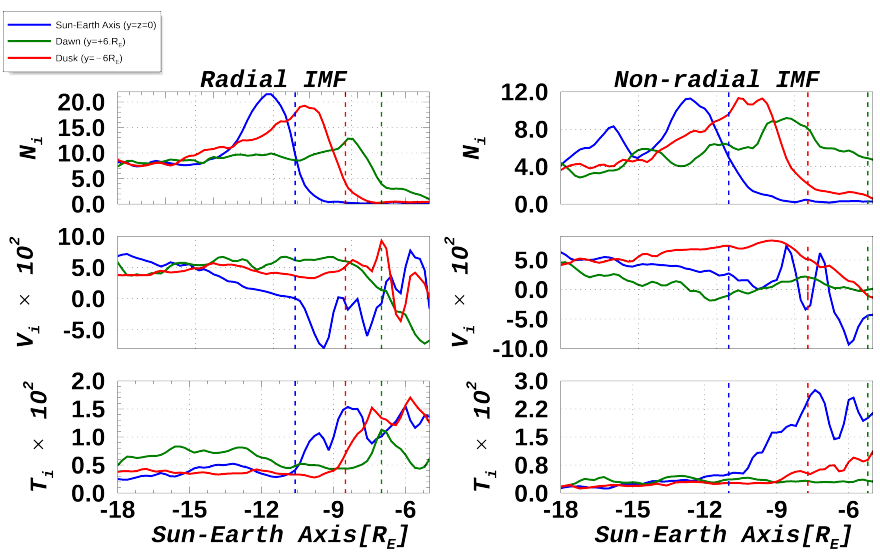

Figure 11. Ion density, velocity, and temperature $\left(N_{i}, V_{i}, T_{i}\right)$ are plotted along the OX-axis and at \pm 6 on both dusk and dawn directions in the XY plane. Vertical dashed lines show the MP position at the subsolar point(blue), dusk flank(red), and dawn flank(green) quantities show dusk-dawn asymmetry at the $\pm 6 R_{E}$. 

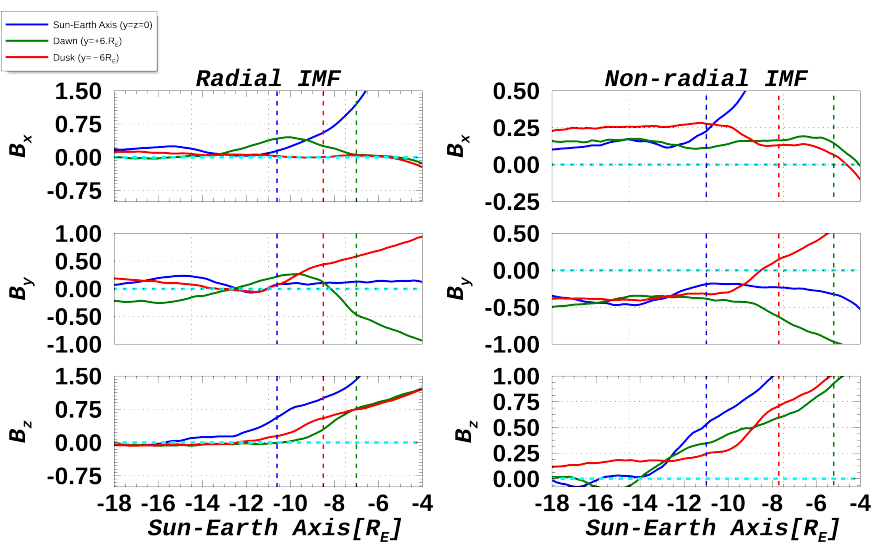

1

Figure 12. IMF components, $B_{x}, B_{y}$, and $B_{z}$ are plotted along the OX-axis and at \pm 6 on both dawn and dusk directions in the XY plane. Vertical dashed lines show the MP position at the subsolar point (blue), dusk flank (red), and dawn flank (green) quantities show dusk-dawn asymmetry at the $\pm 6 R_{E}$. Horizontal cyan dashed line shows the zero value of IMF. Change of IMF polarity is significantly seen in $B_{y}$ component. 